\title{
Unveiling and exemplifying the unitary equivalence of discrete time quantum walk models
}

\author{
B F Venancio ${ }^{1}$, F M Andrade ${ }^{2}$, M G E da Luz ${ }^{1}$ \\ ${ }^{1}$ Departamento de Física, Universidade Federal do Paraná, C.P. 19044, 81531-980 \\ Curitiba-PR, Brazil \\ ${ }^{2}$ Departamento de Matemática e Estatística, Universidade Estadual de Ponta \\ Grossa, 84030-900 Ponta Grossa-PR, Brazil \\ E-mail: bfvenancio@fisica.ufpr.br, fmandrade@uepg.br, \\ luz@fisica.ufpr.br (corresponding author)
}

\begin{abstract}
The two major discrete time formulations for quantum walks, coined and scattering, are unitarily equivalent for arbitrary position dependent transition amplitudes and any topology (PRA 80, 052301 (2009)). Although the proof explicit describes the mapping obtention, its high technicality may hinder relevant physical aspects involved in the equivalence. Discussing concrete examples - the most general constructions for the line, square and honeycomb lattices - here we unveil the similarities and differences of these two versions of quantum walks. We moreover show how to derive the dynamics of one from the other by means of proper projections. We perform calculations for different probability amplitudes like, Hadamard, Grover, Discrete Fourier Transform and the uncommon in the area (but interesting) Discrete Hartley Transform, comparing the evolutions. Our study illustrates the models interplay, an important issue for implementations and applications of such systems.
\end{abstract}

PACS numbers: 03.67.Lx,05.40.Fb

Submitted to: J. Phys. A: Math. Gen. 


\section{Introduction}

Since their introduction [1, 2, 3], quantum walks (QWs), a paradigmatic and relatively simple class of quantum systems [4], have found many applications in different areas of science (see, e.g., the recent review in [5]). At a first glance, such usefulness may be attributed to QWs distinct possible formulations. For instance, although in all cases the dynamics take place in discrete spatial structures, graphs (or lattices), time can be either a continuous [6] or a discrete variable. Moreover, in discrete time versions, the evolution can be dictated by inner states called "coins" [7] (coined QWs, CQWs) or resulting from scattering-like processes [8, 9, 10] (scattering QWs, SQWs).

Regarding usages for QWs, some aspects of the general searching problem [11] seem to be very appropriate for SQWs [12], including identification and comparison of parts of a graph [13, 14]. Also, SQWs are frequently considered in the study of scattering in semi-infinite graphs [9, 10, 15], a relevant configuration to implement universal quantum computation through QWs [16]. On the other hand, the literature proposes a much larger number of applications for CQWs [5, 17, 18, 19]. Examples are: transport in biological systems [20] (in a continuous time context, see [21]); BoseEinstein condensates redistribution [22]; quantum phase transition in optical lattices [23]; decoherence processes [24]; and even quantum games [25]. In particular, CQWs are widely discussed in quantum computation as a tool for the development of quantum algorithms [26].

As it concerns implementations, distinct experimental setups based on distinct physical phenomena can be used to actually build QWs. For CQWs, protocols based on trapped atoms in optical lattices [27], quantum dots [28], photons orbital angular momentum [29], and QED cavities [30], to cite just a few, have been devised. Concrete lab realizations were constructed with waveguide lattices [31] (refers to [32] for waveguides in the continuous time case), passive optical elements [33], and liquid-state nuclear magnetic resonance [34, again only mentioning few examples (an overview is given in [35]). By their turn, SQWs could be associated to optical networks [36, 37]

and eventually may be ensemble just with linear optical elements [12, 13], similarly as done in [38] to fabricate a quantum version of Galton's quincunx (a classical mechanical machine which at certain locations "chooses" - with 50\%:50\% probability - between two directions to go for a traveling ball).

The above mentioned models distinctions for applications (and in a less extend for implementations) are, however, due to practical and operation instead to fundamental reasons. Indeed, continuous time and coined QWs are closely related, since they lead to a same dynamics in proper limits [39, 40] (although the limits may not be so direct to achieve [40]). Furthermore, it has been rigorously proven that CQWs and SQWs are unconditionally (i.e., for arbitrary topologies and spatially dependent transition probabilities) unitary equivalent [41].

These kindred relations open important perspectives in the employment of QWs, specially for the discrete time versions. They should be equally appropriate in any 
application and in principle (see comments in Sec. 4), a same physical implementation would be capable to simulate both CQWs and SQWs. But to benefit from such connections, it is necessary a clear understanding of similarities and differences of the two system versions as well as the correct way to obtain one from another. The proof in [41, having as the main goal to formally demonstrate their equivalence, relies on general and very technical arguments, making difficult to fully appreciate the conceptual features associated to the QWs reciprocities. Thus, knowing that CQWs and SQWs are always equivalent, we can focus on representative cases, discussing in details the interplay between the discrete time formulations. Hence, here we illustrate that different QWs are in fact closely tied and a specific model choice might be much more a matter of practicality than of unfeasibility of the corresponding sibling construction.

The work is organized as the following. From a parallel with usual classical random walks, in Sec. 2 we discuss for the 1D case the main characteristics of CQWs and SQWs, also addressing their mapping. We do so assuming the most general situation of complete arbitrary position dependent transition probabilities at the 1D lattice sites. In Sec. 3, the same type of analysis is carried out for two 2D lattices, the square and the honeycomb. The models are formulated in details and simulations, exemplifying their distinctions for the time evolution along the graphs structures, are presented. Importantly, we show how the spatial probabilities distributions for one model can be obtained from the other through proper projections. Finally, results for different coin (and the equivalent scattering) matrices operators are compared. It includes the more common Hadamard, Grove and Discrete Fourier Transform, as well as the not so usual (but interesting) Discrete Hartley Transform and few others. A short conclusion is drawn in Sec. 4.

\section{Discrete quantum walks on the line: two different views}

To better understand the main ideas underlying the construction of the existing discrete QWs models, we first recall an interesting way to view the classical case on the line (1D).

Consider a classical walker, starting in $x=0$ at $t=0$, that takes steps of fixed length $L$ and moves with constant speed $v$. Its simple dynamics is described as the following. Each time the walker reaches a position $x(t=n \tau)=x_{n}= \pm j L(j=0,1, \ldots)$ for $n=0,1, \ldots$ and $\tau=L / v$, it randomly (and instantaneously) chooses a new direction to go, either to the right $(\sigma=+1)$, with probability $p$, or to the left $(\sigma=-1)$, with probability $1-p$. So, this $1 \mathrm{D}$ continuous system can be characterized by two processes taking place in an "effective lattice": (i) one purely stochastic (choosing directions), occurring at the "lattice sites" $\pm j L$; and (ii) other purely deterministic (ballistic motion, with $x(t)=x_{n-1}+\sigma v\left(t-t_{n-1}\right)$ for $\left.(n-1) \tau \leq t \leq n \tau\right)$, occurring along the "lattice bonds". Such view is schematically represented in Fig. 1.

The construction of a discrete time QW closely follows the previous picture. Essentially, there are two possible implementations, based on what we assume as the primary process, either (i) or (ii) above, to describe the quantum states. Indeed, in the 




Figure 1. (a) A classical random walk faced as alternate stochastic and deterministic processes along a 1D lattice. (b) Example of a possible path for $t=6 \tau$, whose probability is $p^{2}(1-p)^{4}$. Here, $p[1-p]$ is the probability to go to right [left].

(a)

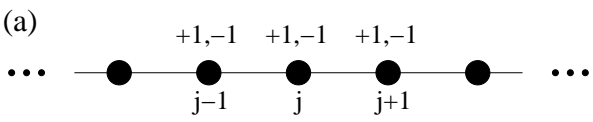

(b)

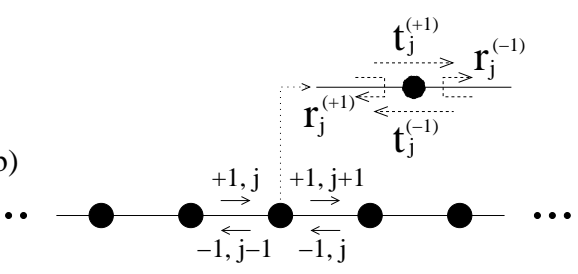

Figure 2. For QWs in 1D, the associated "Hilbert lattice", but which is not necessarily a spatial structure once the states do not need to be position eigenvectors. The states are defined (a) on the sites, coined, and (b) along the bonds, scattering, formulations. For SQWs, details in (b) show the scattering quantum amplitudes defined on each site.

first case (associated to CQWs) the quantum states describe the system at the lattice site positions: classically, the locations where it is made a probabilistic choice about the next step direction. In the second case (associated to SQWs) the quantum states are defined on the bonds: classically, corresponding to the deterministic locomotion along the lattice. This is an important distinction since each model has a different state representation with a different possible interpretation.

One does not need to relate any "lattice" structure to QWs. Nevertheless, it is instructive to go on with such analogy and to view quantum walks in 1D as a dynamics defined on a "Hilbert lattice" [42], depicted in Fig. 2. In this way, we can associate the classical step length $L$ to the characteristic unit distance $\Delta j=1$ between two consecutive "sites" of the Hilbert lattice. Moreover, in both cases the discrete time evolution is given by a single step unitary operator $U$, acting on states belonging to this Hilbert (lattice) space, so that $|\Psi(n+1)\rangle=U|\Psi(n)\rangle$. 


\subsection{The coined quantum walk model}

Quantum states are defined on the sites j, Fig. 2 (a). Then, we consider the basis states $\{|j\rangle\}$, which span the Hilbert subspace $\mathcal{H}_{p}$. Moreover, to any $j$ we associate an inner two-level Hilbert subspace $\mathcal{H}_{c}$, whose "coin" states are $| \pm\rangle$. They give the correct unitary "stochastic" character for the problem [2,43. Also, their role is somehow similar to that of choosing directions in the classical case: once $|j\rangle$ represents only spatial "location" (sites), then "direction" must be played by the auxiliary coin states.

The appropriate orthonormal basis to describe the entire space $\mathcal{H}=\mathcal{H}_{p} \otimes \mathcal{H}_{c}$ $\left(L^{2}(\mathbb{Z}) \otimes L^{2}\left(\mathbb{Z}_{2}\right)\right)$ is $\{|j\rangle \otimes|\sigma\rangle\}$, with $\sigma= \pm$ and $j=0, \pm 1, \pm 2, \ldots$. Defining a shift operator as $S|j\rangle=|j+1\rangle$ (and $S^{\dagger}|j\rangle=|j-1\rangle$ ), we write

$$
U_{c}=\left(S \otimes|+\rangle\left\langle+\left|+S^{\dagger} \otimes\right|-\right\rangle\langle-|\right)\left(\sum_{j}|j\rangle\langle j| \otimes C^{(j)}\right) .
$$

$U_{c}$ is unitary if the coin-flip operators $C^{(j)}$ 's are also unitary. There are many possible choices for the $C$ 's 44, a common one being the Hadamard's, for which $\forall j, C^{(j)}=H_{2}$ with $H_{2}|\sigma\rangle=(|-\sigma\rangle-\sigma|\sigma\rangle) / \sqrt{2}$. However, the most general case is to consider the matrix elements $\left\langle\sigma^{\prime \prime}\left|C^{(j)}\right| \sigma^{\prime}\right\rangle=c_{\sigma^{\prime \prime} \sigma^{\prime}}^{(j)}$ and to assure unitarity just by imposing

$$
\begin{aligned}
& \left|c_{+++}^{(j)}\right|^{2}+\left|c_{-+}^{(j)}\right|^{2}=\left|c_{--}^{(j)}\right|^{2}+\left|c_{+-}^{(j)}\right|^{2}=1, \quad\left|c_{+-}^{(j)}\right|^{2}=\left|c_{-+}^{(j)}\right|^{2}, \\
& c_{++}^{(j)}\left[c_{-+}^{(j)}\right]^{*}+c_{+-}^{(j)}\left[c_{--}^{(j)}\right]^{*}=0 .
\end{aligned}
$$

Thus, we have

$$
U_{c}|j\rangle \otimes|\sigma\rangle=c_{\sigma \sigma}^{(j)}|j+\sigma\rangle \otimes|\sigma\rangle+c_{-\sigma \sigma}^{(j)}|j-\sigma\rangle \otimes|-\sigma\rangle .
$$

One of the most important applications of CQWs is in quantum computation. So, it is usual to define the above matrix elements in a way easy to associate to quantum

gate operators. It explains, e.g., the frequent choice in the literature of the Hadamard's coin (fundamental to manipulate q-bits). This practice, however, leads to constructions of coin matrices in a format not common among physicists, but popular in mathematics and computer science (as nicely explained and exemplified in Ref. [45], e.g., p. xxiii). Indeed, the states $| \pm\rangle$ are written as the column matrices

$$
|-\rangle=\left(\begin{array}{c}
1 \\
0
\end{array}\right), \quad|+\rangle=\left(\begin{array}{l}
0 \\
1
\end{array}\right),
$$

opposite to the usual spin-up and spin-down convention. Following this representation, the most general form for the coin operator, obeying to Eq. (2) and having the Hadamard as a particular case is (see also [46, 47, 48])

$$
C^{(j)}=\exp \left[i \gamma_{j}\right]\left(\begin{array}{cc}
\exp \left[i \xi_{j}\right] \cos \left[\theta_{j}\right] & \exp \left[i \zeta_{j}\right] \sin \left[\theta_{j}\right] \\
\exp \left[-i \zeta_{j}\right] \sin \left[\theta_{j}\right] & -\exp \left[-i \xi_{j}\right] \cos \left[\theta_{j}\right]
\end{array}\right) .
$$

with $0 \leq \gamma_{j}, \zeta_{j}, \xi_{j}, \theta_{j}<2 \pi$. Then, Eq. (3) reads

$$
\begin{aligned}
U_{c}|j\rangle \otimes|\sigma\rangle= & -\sigma \exp \left[i\left(\gamma_{j}-\sigma \xi_{j}\right)\right] \cos \left[\theta_{j}\right]|j+\sigma\rangle \otimes|+\sigma\rangle \\
& +\exp \left[+i\left(\gamma_{j}+\sigma \zeta_{j}\right)\right] \sin \left[\theta_{j}\right]|j-\sigma\rangle \otimes|-\sigma\rangle .
\end{aligned}
$$




\subsection{The scattering quantum walk model}

For our purposes, it is easier to formulate the scattering model in a slightly different (but completely equivalent) way than usually done in the literature [10]. Along each bond, joining two consecutive sites, say $j-1$ and $j$ (see Fig. 2 (b)), we assume two possible states $|+1, j\rangle$ and $|-1, j-1\rangle$. Hence, contrasting to the coined model, now the quantum number $\sigma= \pm 1$ is associated to a "direction" along a bond in the Hilbert lattice. The full Hilbert space $\mathcal{H}$ is no longer a direct product of two subspaces. Each basis element $|\sigma, j\rangle$ belongs to $L^{2}\left(\mathbb{Z} \times \mathbb{Z}_{2}\right)$ and satisfies to $\left\langle j^{\prime}, \sigma^{\prime} \mid \sigma, j\right\rangle=\delta_{j^{\prime} j} \delta_{\sigma^{\prime} \sigma}$.

Defining the operators $T$ and $R$ by

$$
T|\sigma, j\rangle=t_{\sigma}^{(j)}|\sigma, j+\sigma\rangle, R|\sigma, j\rangle=r_{\sigma}^{(j)}|-\sigma, j-\sigma\rangle,
$$

and

$$
T^{\dagger}|\sigma, j\rangle=t_{\sigma}^{(j-\sigma)^{*}}|\sigma, j-\sigma\rangle, R^{\dagger}|\sigma, j\rangle=r_{-\sigma}^{(j-\sigma)^{*}}|-\sigma, j-\sigma\rangle,
$$

the one step time evolution is simply

$$
U_{s}=T+R,
$$

so that

$$
U_{s}|\sigma, j\rangle=t_{\sigma}^{(j)}|\sigma, j+\sigma\rangle+r_{\sigma}^{(j)}|-\sigma, j-\sigma\rangle .
$$

The unitarity of $U_{s}$ implies [49]

$$
\left|t_{\sigma}^{(j)}\right|^{2}+\left|r_{\sigma}^{(j)}\right|^{2}=1, \quad\left|r_{\sigma}^{(j)}\right|^{2}=\left|r_{-\sigma}^{(j)}\right|^{2}, \quad r_{-\sigma}^{(j)} t_{\sigma}^{(j)^{*}}+r_{\sigma}^{(j)^{*}} t_{-\sigma}^{(j)}=0,
$$

which are exactly the relations satisfied by the reflection and transmission amplitudes in a quantum scattering problem in 1D [49, 50], resulting from the unitarity of the $S$ scattering matrix.

Equation (11) automatically holds if for any $j$ (with $0 \leq \rho_{j} \leq 1$ and $0 \leq \lambda_{j}, \phi_{j}, \varphi_{j}<$ $2 \pi)$

$$
\begin{aligned}
t_{\sigma}^{(j)} & =\exp \left[i \lambda_{j}\right]\left(\sqrt{1-\rho_{j}} \exp \left[i \sigma \phi_{j}\right]\right), \\
r_{\sigma}^{(j)} & =\exp \left[i \lambda_{j}\right]\left(\sigma \sqrt{\rho_{j}} \exp \left[i \sigma \varphi_{j}\right]\right) .
\end{aligned}
$$

If $\lambda_{j}=\lambda$ for all $j$, without loss of generality we can set $\lambda=0$. We should mention that the expressions in Eq. (12) are not the only possibility [42]. There is a certain arbitrariness in signals convention. The present choice, however, has a direct physical motivation. For time-reverse invariant systems $t_{+}^{(j)}=t_{-}^{(j)}$ [50]. Then, if we also assume real amplitudes, the signals for the $r$ 's must be opposite, exactly the case in Eq. (12). Furthermore, for $j$-independent scattering coefficients, the above (with $\phi_{j}=\varphi_{j}=0$ ) are the relations adopted in the original work introducing SQW models [8].

\subsection{Obtaining the probabilities}

At this point we should emphasize that the above constructions are more general than simply to quantize the dynamics of a common classical random walk. Here, by common 
we mean that each time a new direction needs to be chosen, we use the same probabilities $(p$ and $1-p)$ to decide between right and left. Quantum mechanically, to allow the parameters to depend on $j$ (cf., Eqs. (5) and (12)) implies that we explicit assume position dependent probability amplitudes. Obviously, by making such parameters $j$ independent, we recover the relation with the usual classical case.

Now, suppose we shall determine which is the probability $P^{(j)}(n)$ to be in the "position" state $j$ (which means a site (bond) state in the coin (scattering) model) at time $n$, regardless the value of the coin (direction) quantum number $\sigma$. We define then

$$
\mathcal{P}_{c}^{(j)}=|j\rangle\left\langle j\left|\otimes \sum_{\sigma}\right| \sigma\right\rangle\left\langle\sigma\left|, \quad \mathcal{P}_{s}^{(j)}=\sum_{\sigma}\right| \sigma, j+\frac{\sigma-1}{2}\right\rangle\left\langle j+\frac{\sigma-1}{2}, \sigma\right|,
$$

respectively, the coin and scattering position projector operators. The sought probability is the expected value

$$
P^{(j)}(n)=\left\langle\Psi(n)\left|\mathcal{P}^{(j)}\right| \Psi(n)\right\rangle,
$$

for $\mathcal{P}^{(j)}$ one of the expressions in Eq. (13).

Projection is thus an essential ingredient in defining a QW model, which differs from classical walk systems by the typical interference effects due to the Eq. (14) (see, e.g., Ref. [51]).

\subsection{The unitary equivalence of the two $1 D$ quantum walk models}

Finally, in the present 1D topology the unitary equivalence between CQWs and SQWs is straightforwardly established.

First, note the one-to-one correspondence between their full Hilbert spaces, $L^{2}(\mathbb{Z}) \otimes$ $L^{2}\left(\mathbb{Z}_{2}\right) \equiv L^{2}\left(\mathbb{Z} \times \mathbb{Z}_{2}\right)$. So, it follows directly the existence of an isomorphic unitary operator [8] $E: \mathcal{H} \rightarrow \mathcal{H}$, given by

$$
E|\sigma, j\rangle=|j\rangle \otimes|\sigma\rangle, \quad E^{\dagger}|j\rangle \otimes|\sigma\rangle=|\sigma, j\rangle .
$$

Second, (i) inspecting Eqs. (3) and (10); (ii) considering the most general conditions for the models quantum coefficients, represented by Eqs. (2) and (11) (observe that Eqs. (5) and (12), more usual in the literature, already obey particular conventions); and (iii) taking into account Eq. (15); one finds that the following coefficients play complete similar roles

$$
c_{\sigma \sigma}^{(j)} \leftrightarrow t_{\sigma}^{(j)} \quad \text { and } \quad c_{-\sigma \sigma}^{(j)} \leftrightarrow r_{\sigma}^{(j)} .
$$

Hence, setting $c_{\sigma \sigma}^{(j)}=t_{\sigma}^{(j)}$ and $c_{-\sigma \sigma}^{(j)}=r_{\sigma}^{(j)}$ for all $j$, we have in both models exactly the same probability amplitudes for their time evolutions.

Third, from Eqs. (3), (10), (15) and assuming the above equalities, the resulting dynamics are unitary equivalent once

$$
U_{s}=E^{\dagger} U_{c} E
$$

As it is known 8], even when different QW models are unitary equivalent, the associated probabilities - obtained through direct projections - can be distinct. This 
is so because we are choosing contrasting physical representations, sites (coined) and bonds (scattering), to describe the problem. The specific states which characterize one of these "spatial" configurations are not akin to the states for the other. For instance, the two states corresponding to the site $j$ in CQWs, $|j\rangle \otimes|\sigma= \pm\rangle$, are mapped to states at different bonds in SQWs. Therefore, the probability to be in a unique site is not equal to the probability to be in a unique bond (cf., Eqs. (13)-(14)). Mathematically, it is related to the fact that considering Eqs. (13) and (15), we get $\mathcal{P}_{s}^{(j)} \neq E^{\dagger} \mathcal{P}_{c}^{(j)} E$ and $\mathcal{P}_{c}^{(j)} \neq E \mathcal{P}_{s}^{(j)} E^{\dagger}$ (inequalities holding true in any topology given the results in [41]). Thus, for $|\Psi\rangle_{s}$ and $|\Psi\rangle_{c}=E|\Psi\rangle_{s}$, we have from Eq. (14) that generally $P_{s}^{(j)} \neq P_{c}^{(j)}$.

Nevertheless, due to the QWs unitary equivalence we can recover the probabilities from each other version by constructing proper cross projector operators. Indeed, defining

$$
\left.\mathcal{P}_{s}^{(j)}\right|_{c}=E^{\dagger} \mathcal{P}_{c}^{(j)} E,\left.\quad \mathcal{P}_{c}^{(j)}\right|_{s}=E \mathcal{P}_{s}^{(j)} E^{\dagger},
$$

one readily obtains the probabilities for the coined (scattering) model by applying $\left.\mathcal{P}_{s}^{(j)}\right|_{c}$ $\left(\left.\mathcal{P}_{c}^{(j)}\right|_{s}\right)$ to the state $|\Psi(n)\rangle_{s}\left(|\Psi(n)\rangle_{c}\right)$, evolved according to the scattering (coined) formulation.

The numerical examples for 2D lattices in Section 3 will clearly illustrate all the above observations.

\section{More general topologies: two illustrative examples}

As already mentioned, it has been proven in Ref. 41] that CQWs and SQWs are always related by an unitary transformation. In the previous Section, profiting from its relative simplicity (although considering the most general situation, hence extending the results of Ref. [8]), we have explicit illustrated so for the 1D case.

However, the benefits in being able to map CQWs and SQWs become really evident when one considers more complex topologies. Thus, next we present a detailed analysis of the correspondence between CQWs and SQWs in two particular, but very instructive, 2D examples, square and honeycomb lattices.

\subsection{Quantum walks on a square lattice}

The square lattice, represented in Fig. 3, is the natural 2D extension of the 1D topology. In the following we describe in such a case the coined and scattering QW formulations, as well as their unitary equivalence.

\subsubsection{The coined formulation}

In Fig. 3 (a) we schematically represent the coined Hilbert lattice, whose basis states are $\{|j\rangle \otimes|k\rangle \otimes|\sigma\rangle\}$, with $\sigma=1,2,3,4$ and $j, k=0, \pm 1, \pm 2, \ldots$. For notation simplicity, we do not write the "spatial" states in the form $|j\rangle_{x} \otimes|k\rangle_{y}$ (and operators as $\left.A_{x} \otimes B_{y}\right)$ to distinguish between the two distinct 1D dimensions. Also, we assume the natural ordering convention $(A \otimes B \otimes C)|\alpha\rangle \otimes|\beta\rangle \otimes|\gamma\rangle=A|\alpha\rangle \otimes B|\beta\rangle \otimes C|\gamma\rangle$. 


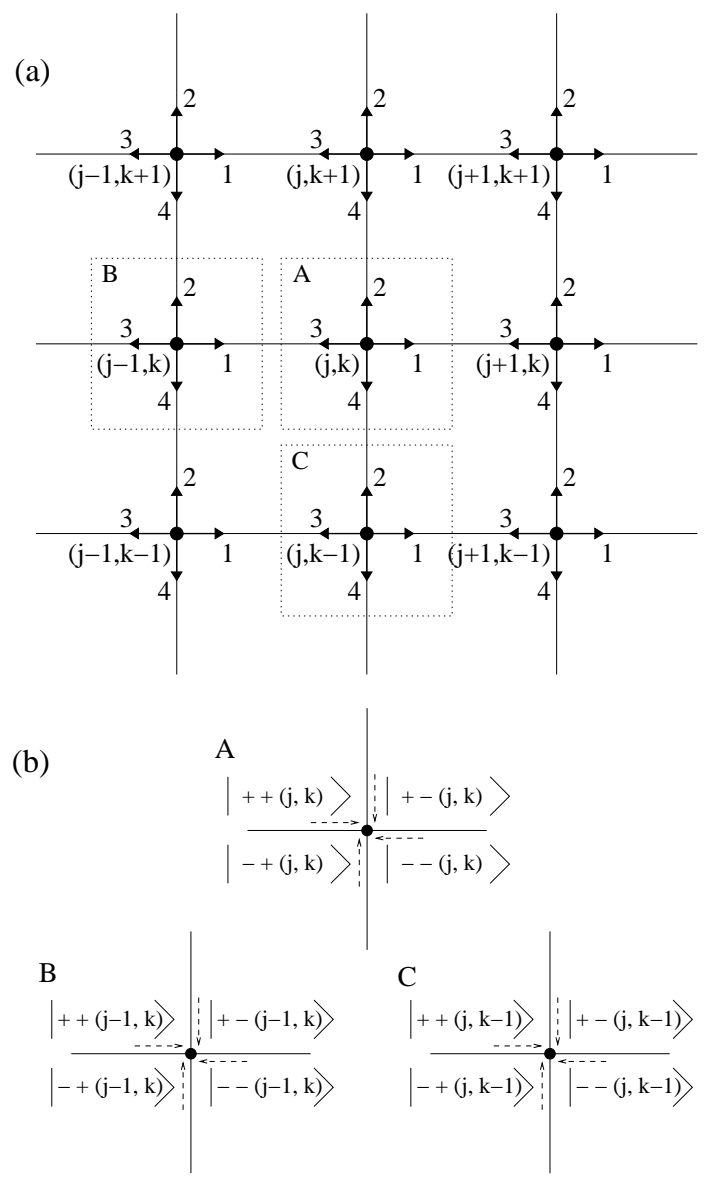

Figure 3. A QW on a square lattice. (a) At each site $(j, k)$, the arrows schematically represent the four inner states, $\sigma=1,2,3,4$, of the coined formulation. (b) For the scattering formulation, it is shown the four "incoming" states propagating to the lattice sites marked in (a). The two possible states defined on the bond common to the regions $\mathrm{A}$ and $\mathrm{B}(\mathrm{A}$ and $\mathrm{C})$ are $|++,(j, k)\rangle$ and $|--,(j-1, k)\rangle(|-+,(j, k)\rangle$ and $|+-,(j, k-1)\rangle)$.

For the inner coin states $|\sigma\rangle$, written as

$$
|1\rangle=\left(\begin{array}{l}
0 \\
1 \\
0 \\
0
\end{array}\right),|3\rangle=\left(\begin{array}{l}
1 \\
0 \\
0 \\
0
\end{array}\right),|2\rangle=\left(\begin{array}{l}
0 \\
0 \\
0 \\
1
\end{array}\right),|4\rangle=\left(\begin{array}{l}
0 \\
0 \\
1 \\
0
\end{array}\right),
$$

the coin operator matrix (at each $(j, k)$ ) reads

$$
C^{(j, k)}=\left(\begin{array}{cccc}
c_{33}^{(j, k)} & c_{31}^{(j, k)} & c_{34}^{(j, k)} & c_{32}^{(j, k)} \\
c_{13}^{(j, k)} & c_{11}^{(j, k)} & c_{14}^{(j, k)} & c_{12}^{(j, k)} \\
c_{43}^{(j, k)} & c_{41}^{(j, k)} & c_{44}^{(j, k)} & c_{42}^{(j, k)} \\
c_{23}^{(j, k)} & c_{21}^{(j, k)} & c_{24}^{(j, k)} & c_{22}^{(j, k)}
\end{array}\right)
$$

The unitarity of $C$, directly extending the relations in Eq. (2), leads to

$$
\sum_{l} c_{i l}^{(j, k)} c_{m l}^{(j, k)^{*}}=\sum_{l} c_{l i}^{(j, k)} c_{l m}^{(j, k)^{*}}=\delta_{i m} .
$$




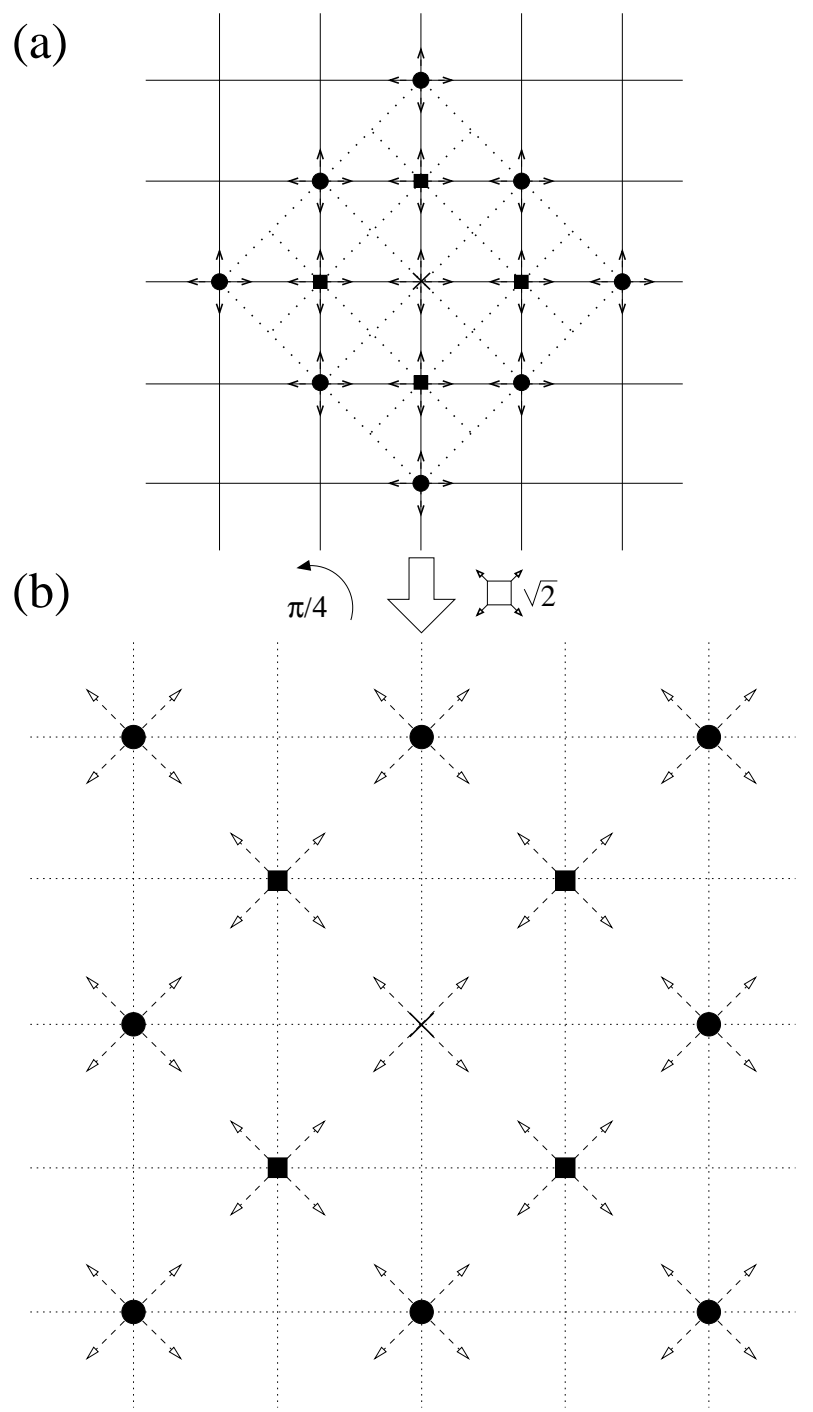

Figure 4. Schematics of the one step time evolution for the coined model in a square lattice in the natural (a) and the diagonal (b) cases. The system, initially at the state represented by the site $\times$, in a first step can reach the states represented by the sites -. In the second step, the states which can be visited are those represented by the sites $\bullet$ and $\times$. The diagonal version (dotted lattices) can be thought as the natural one rotated by $\pi / 4$ and rescaled by a factor $\sqrt{2}$.

As the one step time evolution, there are two common possibilities. The first is the so called natural choice, since single steps displacements follow the natural topology of the lattice [52], i.e., from a given site representing a state, it can go either to right, left, up or down [7, 53] (Fig. 4 (a)). The second is the diagonal version [54]. In this case, single steps are those in which "moves" take place along the diagonals of the Hilbert lattice (Fig. 4 (b)).

Let us define (for $S$ as in Sec. 2-1)

$$
N=S \otimes \mathbb{I} \otimes|1\rangle\left\langle 1\left|+S^{\dagger} \otimes \mathbb{I} \otimes\right| 3\right\rangle\langle 3|+\mathbb{I} \otimes S \otimes| 2\rangle\left\langle 2\left|+\mathbb{I} \otimes S^{\dagger} \otimes\right| 4\right\rangle\langle 4|,(22)
$$

with $\mathbb{I}$ the identity operator. The time evolution for the natural version, an extension 
of Eq. (1), yields

$$
U_{c}=N \times\left(\sum_{j, k}|j\rangle\langle j|\otimes| k\rangle\langle k| \otimes C^{(j, k)}\right) .
$$

For the diagonal version, we first define the operator

$$
D=\mathbb{I} \otimes S \otimes|1\rangle\left\langle 1\left|+\mathbb{I} \otimes S^{\dagger} \otimes\right| 3\right\rangle\left\langle 3\left|+S^{\dagger} \otimes \mathbb{I} \otimes\right| 2\right\rangle\langle 2|+S \otimes \mathbb{I} \otimes| 4\rangle\langle 4| .(24)
$$

So, the time evolution is given by

$$
U_{c-\text { diagonal }}=D U_{c} \text {. }
$$

Equation (25), with $D$ as in Eq. (24), has a very simple interpretation (if we maintain that Eq. (25) represents a single step). Indeed, the diagonal case is essentially the natural one rotated by $\pi / 4$ (anti-clockwise) in the Hilbert lattice. Moreover, quantities associated to any norm (length scale) defined on the lattice, e.g., diffusiveness, should be re-scaled by a factor $\sqrt{2}$, which is just the ratio between the diagonal and side of the unitary cell, Fig. 4. In all our further analysis, we will consider only the natural version, observing that the diagonal would easily follow from Eq. (25).

Finally, for the natural coined model the projector operator is

$$
\mathcal{P}_{c}^{(j, k)}=|j\rangle\langle j|\otimes| k\rangle\left\langle k\left|\otimes \sum_{\sigma=1}^{4}\right| \sigma\right\rangle\langle\sigma| .
$$

\subsubsection{The scattering model}

For $\sigma_{x}, \sigma_{y}= \pm 1$ ( \pm for short) and $j, k=0, \pm 1, \pm 2, \ldots$, the scattering model basis states are $\left\{\left|\sigma_{x} \sigma_{y},(j, k)\right\rangle\right\}$, Fig. $3(\mathrm{~b})$. The time evolution is again $U_{s}=T+R$, with

$$
\begin{aligned}
& T\left|\sigma_{x} \sigma_{y},(j, k)\right\rangle=\sum_{\{\alpha, \beta\} \neq\left\{-\sigma_{x},-\sigma_{y}\right\}} t_{\alpha \beta, \sigma_{x} \sigma_{y}}^{(j, k)}\left|\alpha \beta,\left(j+\frac{\alpha|\alpha+\beta|}{2}, k+\frac{\beta|\alpha-\beta|}{2}\right)\right\rangle, \\
& R\left|\sigma_{x} \sigma_{y},(j, k)\right\rangle=r_{-\sigma_{x}-\sigma_{y}, \sigma_{x} \sigma_{y}}^{(j, k)}\left|-\sigma_{x}-\sigma_{y},\left(j-\frac{\sigma_{x}\left|\sigma_{x}+\sigma_{y}\right|}{2}, k-\frac{\sigma_{y}\left|\sigma_{x}-\sigma_{y}\right|}{2}\right)\right\rangle .
\end{aligned}
$$

The corresponding actions of $T^{\dagger}$ and $R^{\dagger}$ are a straightforward generalization of Eq. (8), in a format similar to Eq. (27). Defining



the unitarity of $U_{s}$ implies in the unitarity of the scattering matrices $\Gamma^{(j, k)}$ (once more a direct extension of previous results, in this case of Eq. (11)). So, the elements of $\Gamma^{(j, k)}$ must satisfy to relations completely analog to those in Eq. (21). 
For the projector operators, we need to distinguish between the horizontal $\left(\sigma_{x} \times \sigma_{y}=\right.$ $+)$ and the vertical $\left(\sigma_{x} \times \sigma_{y}=-\right)$ bonds. Hence, we define

$$
\begin{aligned}
& \mathcal{P}_{s}^{(j, k ;+)}=\sum_{\sigma_{x}}\left|\sigma_{x} \sigma_{x},\left(j+\frac{\sigma_{x}-1}{2}, k\right)\right\rangle\left\langle\left(j+\frac{\sigma_{x}-1}{2}, k\right), \sigma_{x} \sigma_{x}\right|, \\
& \mathcal{P}_{s}^{(j, k ;-)}=\sum_{\sigma_{y}}\left|-\sigma_{y} \sigma_{y},\left(j, k+\frac{\sigma_{y}-1}{2}\right)\right\rangle\left\langle\left(j, k+\frac{\sigma_{y}-1}{2}\right),-\sigma_{y} \sigma_{y}\right| .
\end{aligned}
$$

\subsubsection{The mapping}

To map the two models, we first make the following identification between the scattering directions and the inner coin quantum numbers $\left(\sigma_{x} \sigma_{y} \leftrightarrow \sigma\right)$ :

$$
++\leftrightarrow 1,-+\leftrightarrow 2,--\leftrightarrow 3,+-\leftrightarrow 4,
$$

which can be cast as $\sigma=\left(5-\left(2+\sigma_{x}\right) \sigma_{y}\right) / 2$.

Using the procedure in the last Section (or likewise, the rigorous construction in Ref. [41]), i.e., to associate the scattering states "incoming" to a certain site (see Fig. 3 (b)) with the coin states at that site, we can set $E$ as (taking into account Eq. (301)

$$
E\left|\sigma_{x} \sigma_{y}(j, k)\right\rangle=|j\rangle \otimes|k\rangle \otimes\left|\frac{5}{2}-\frac{\left(2+\sigma_{x}\right) \sigma_{y}}{2}\right\rangle .
$$

Furthermore, assuming the coefficients in Eq. (20) equal to those in Eq. (28) (which is consistent with the relation in Eq. (30) ), again we find that the two models are unitary equivalent, since for Eqs. (23), (27) and (31), the relation in Eq. (17) holds.

Finally, to obtain the cross projector operators, we define $\left.\mathcal{P}_{s}^{(j, k)}\right|_{c}$ and $\left.\mathcal{P}_{c}^{(j, k)}\right|_{s}$ as in Eq. (18), for $E$ given by Eq. (31), $\mathcal{P}_{c}^{(j, k)}$ by Eq. (26) , and $\mathcal{P}_{s}^{(j, k)}$ by the appropriate $\mathcal{P}_{s}^{(j, k) ; \pm}$ in Eq. (29).

\subsubsection{Examples}

To illustrate the above general constructions, we analyze some particular cases for the probability amplitudes (see, for instance, Refs. [7, 52, 53]). For simplicity, we suppose all the coin (and therefore the scattering) matrices to be independent on the quantum numbers $j$ and $k$. We choose the following four coin operators (whose corresponding scattering matrices are written in exactly the same form):

(a) $x-y$ decoupled Hadamard

$$
C_{H_{2} \oplus H_{2}}=\left(\begin{array}{cc}
H_{2} & 0 \\
0 & H_{2}
\end{array}\right)=\frac{1}{\sqrt{2}}\left(\begin{array}{cccc}
+1 & +1 & 0 & 0 \\
+1 & -1 & 0 & 0 \\
0 & 0 & +1 & +1 \\
0 & 0 & +1 & -1
\end{array}\right) ;
$$

(b) Full $4 \times 4$ Hadamard

$$
C_{H_{4}}=\frac{1}{2}\left(\begin{array}{cccc}
+1 & +1 & +1 & +1 \\
+1 & -1 & +1 & -1 \\
+1 & +1 & -1 & -1 \\
+1 & -1 & -1 & +1
\end{array}\right)
$$


(c) $4 \times 4$ Grover

$$
C_{G_{4}}=\frac{1}{2}\left(\begin{array}{cccc}
-1 & +1 & +1 & +1 \\
+1 & -1 & +1 & +1 \\
+1 & +1 & -1 & +1 \\
+1 & +1 & +1 & -1
\end{array}\right)
$$

(d) $4 \times 4$ Discrete Fourier Transform (DFT)

$$
C_{D F T_{4}}=\frac{1}{2}\left(\begin{array}{cccc}
+1 & +1 & +1 & +1 \\
+1 & +i & -1 & -i \\
+1 & -1 & +1 & -1 \\
+1 & -i & -1 & +i
\end{array}\right)
$$

We compute $U^{n}|\Psi(0)\rangle$, with $n=20$, for both QW models and the above matrices. For each $(j, k)$ we use proper projectors to calculate the probability $P^{(j, k)}(n=20)=$ $\left\langle\Psi(20)\left|\mathcal{P}^{(j, k)}\right| \Psi(20)\right\rangle$. As $|\Psi(0)\rangle$, we take

$$
\begin{aligned}
& |\Psi(0)\rangle_{s}=\frac{1}{2}[|++(0,0)\rangle+i|--(0,0)\rangle+|-+(0,0)\rangle+i|+-(0,0)\rangle], \\
& |\Psi(0)\rangle_{c}=E|\Psi(0)\rangle_{s}=\frac{1}{2}|0\rangle \otimes|0\rangle \otimes[|1\rangle+i|3\rangle+|2\rangle+i|4\rangle] .
\end{aligned}
$$

The $P^{(j, k)}$ 's are displayed as 3D density plots. So, in each graph the heights in the $z$-axis correspond to the probabilities values. Moreover, here and for the honeycomb lattice in Sec. 3.2, each pair of labels $j$ and $k$ in the $x-y$ plane indicates the $(j, k)$ lattice site spatial location. Thus, for the coined formulation, the $P^{(j, k)}$ 's are marked just over the sites. For the scattering formulation, since the states are defined along the bonds, we mark the $P$ 's exactly over the middle points of the corresponding bonds. Hence, we show the probability patterns of each QW version in its own state representation, but in a way which makes easy to qualitatively compare the two models.

The results for the decoupled Hadamard, full Hadamard, Grove, and DFT operators are presented, respectively, in Figs. (5) to (8). The plots are organized as the following. For the coined formulation, Figs. 5-8 (a) [(b)] show the probabilities obtained from the projectors in Eq. (26) [the projectors $\left.\mathcal{P}_{c}^{(j, k)}\right|_{s}$ ]. On the other hand, for the scattering formulation, Figs. 5-8 (c) $[(\mathrm{d})]$ show the $P^{(j, k)}$ 's from the projectors in Eq. (29) [the projectors $\left.\left.\mathcal{P}_{s}^{(j, k)}\right|_{c}\right]$.

By inspecting Figs. (5)-(8), some points become evident. (i) Due to decoupled form of the transition amplitude matrix, Eq. (32), in Fig. 5 we actually have two independent one dimensional evolutions. Hence, each direction is a good example of the 1D results in Sec. 2. (ii) Despite the fact the two QW versions are completely equivalent by unitary transformations, they lead to distinct probability patterns (compare (a) or (d) with (b) or (c)). Indeed, as already emphasized (for instance, the discussion just after Eq. (17) in Sec. 2.4), the one-to-one correspondence between the two formulations pinpoint a dynamical similarity. However, the states in each case represent different (although correlated) aspects of the Hilbert lattice, thus the distributions of the $P$ 's do not need to coincide. (iii) Moreover, such patterns display richer structures in the 



Figure 5. Quantum walks in the square lattice considering the decoupled Hadamard transition coefficients, Eq. (32). The density plots represent the probabilities to be in the different states (as defined by the projector operators) after $n=20$ time steps for $|\Psi(0)\rangle$ given in the main text. The results are for the: (a) coined, (b) scattering obtained from the coined, (c) scattering, and (d) coined obtained form the scattering, models. The graphs (a) and (d) and (b) and (c) are complete identical.

scattering case. To understand so, assume the simpler 1D lattice, for which the initial state $|+, 0\rangle(E|+, 0\rangle=|0\rangle \otimes|+\rangle)$ evolves one time step. For the SQW (CQW), we get $|\Psi(1)\rangle=r_{+}^{(0)}|-,-1\rangle+t_{+}^{(0)}|+,+1\rangle\left(|\Psi(1)\rangle=c_{-+}^{(0)}|-1\rangle \otimes|-\rangle+c_{++}^{(0)}|+1\rangle \otimes|+\rangle\right)$. Considering the Hilbert lattice picture, note that for the former we have two "neighbor" bond states in the expansion of $|\Psi(1)\rangle$, those "attached" to the site 0 . On the other hand, for the latter the site states composing $|\Psi(1)\rangle$ are $j=-1$ and $j=+1$, but not $j=0$. It illustrates a very typical situation in any topology, namely, the SQW dynamics tends to excite "contiguous" spatial (bond) basis states, whereas CQWs may skip some "successive" (site) basis states. Thus, interference [51] is usually more recurrent in SQWs than in CQWs (due to this difference in the spreading of $|\Psi\rangle$ ), explaining the behavior observed in the plots. (iv) Nevertheless, we can recover the $P^{(j, k)}$ 's from each other model by means of the cross projectors (e.g., SQWs in (b) from CQWs in (a) and CQWs in (d) from SQWs in (c)), since the information about one model is always encoded in the other.

We finally mention we have analyzed the diagonal CQW and its corresponding scattering version. We have obtained one formulation $P$ 's from the other by correctly 
Exemplifying the equivalence of discrete quantum walk models

(a)
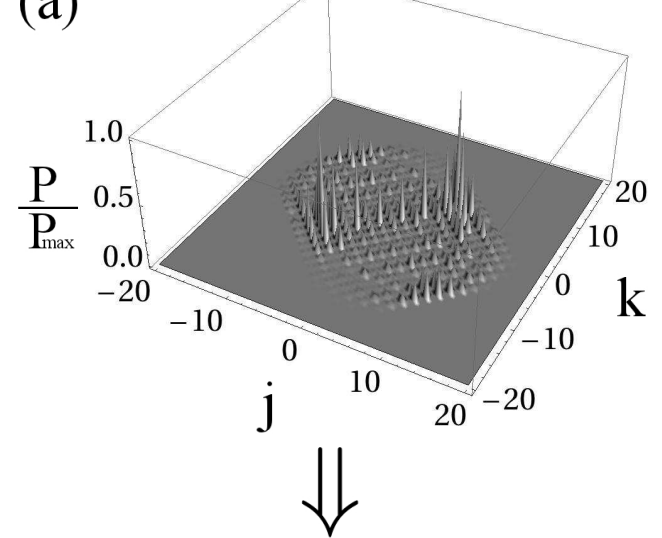

(b)

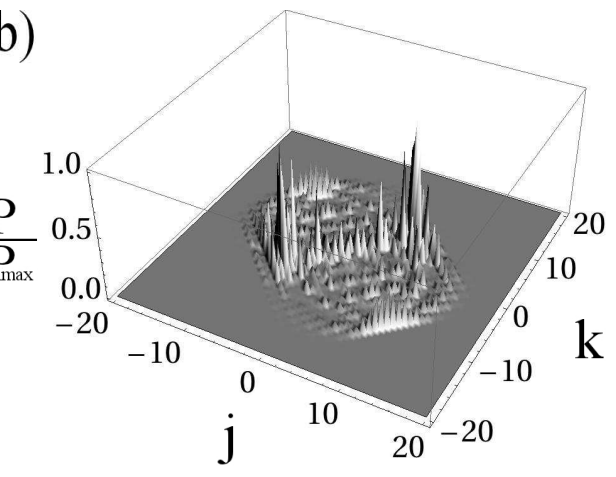

(d)
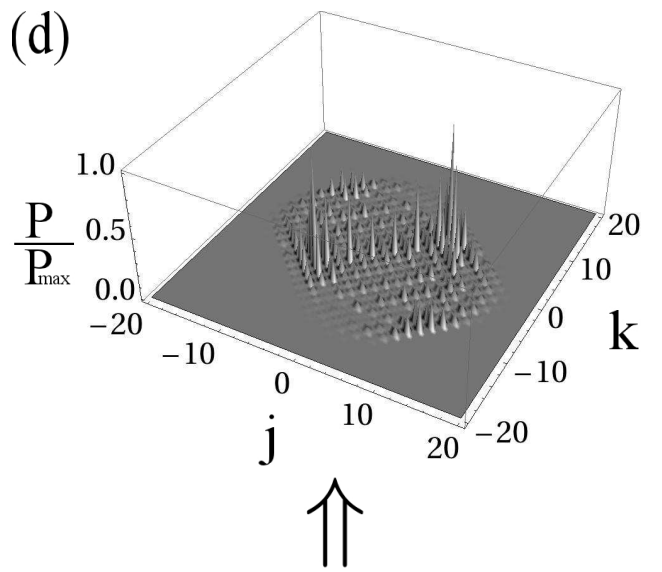

(c)

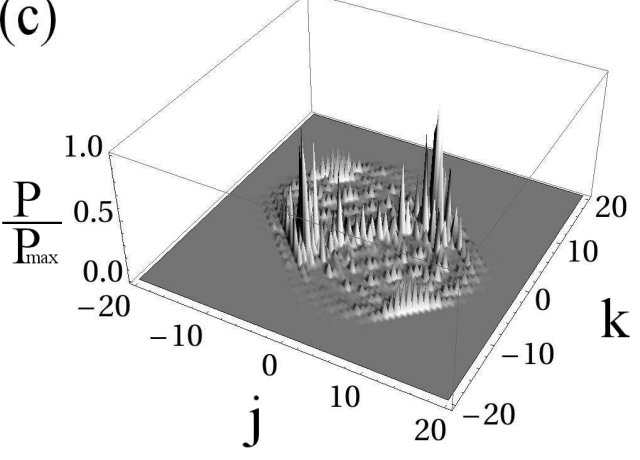

Figure 6. The same as in Fig. 5, but for the $4 \times 4$ Hadamard, Eq. (33).

(a)
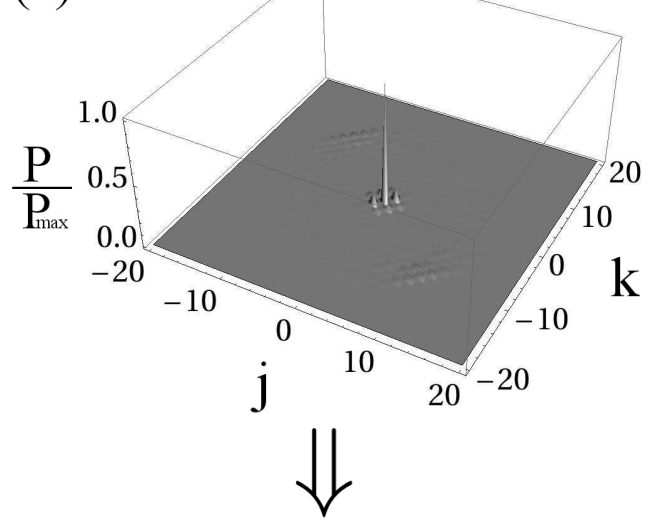

(b)

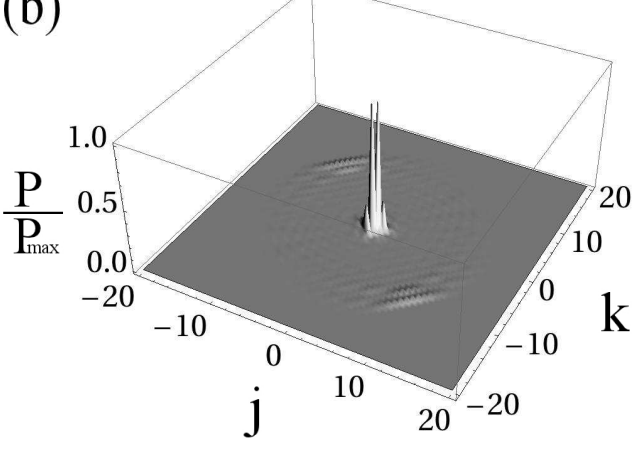

(d)
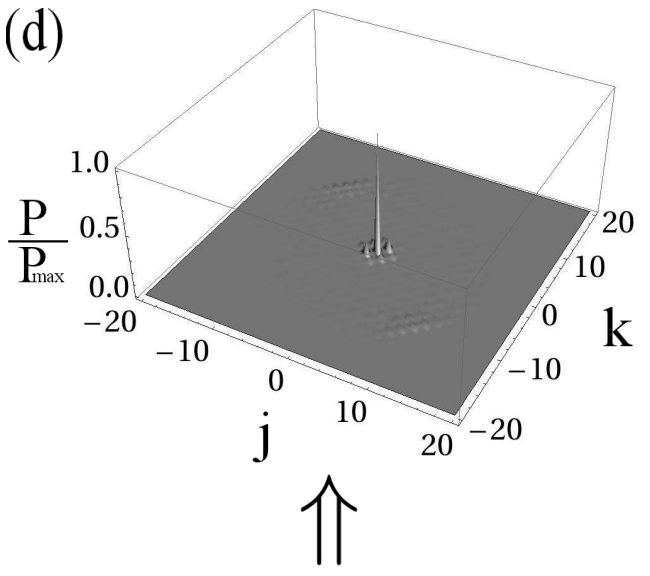

(c)

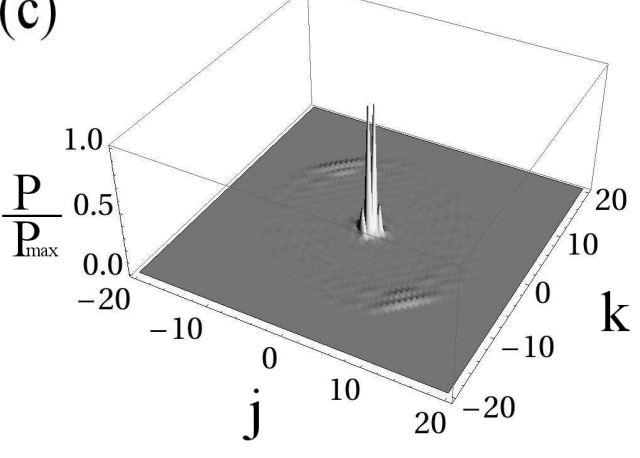

Figure 7. The same as in Fig. 5, but for the $4 \times 4$ Grover, Eq. (34). 

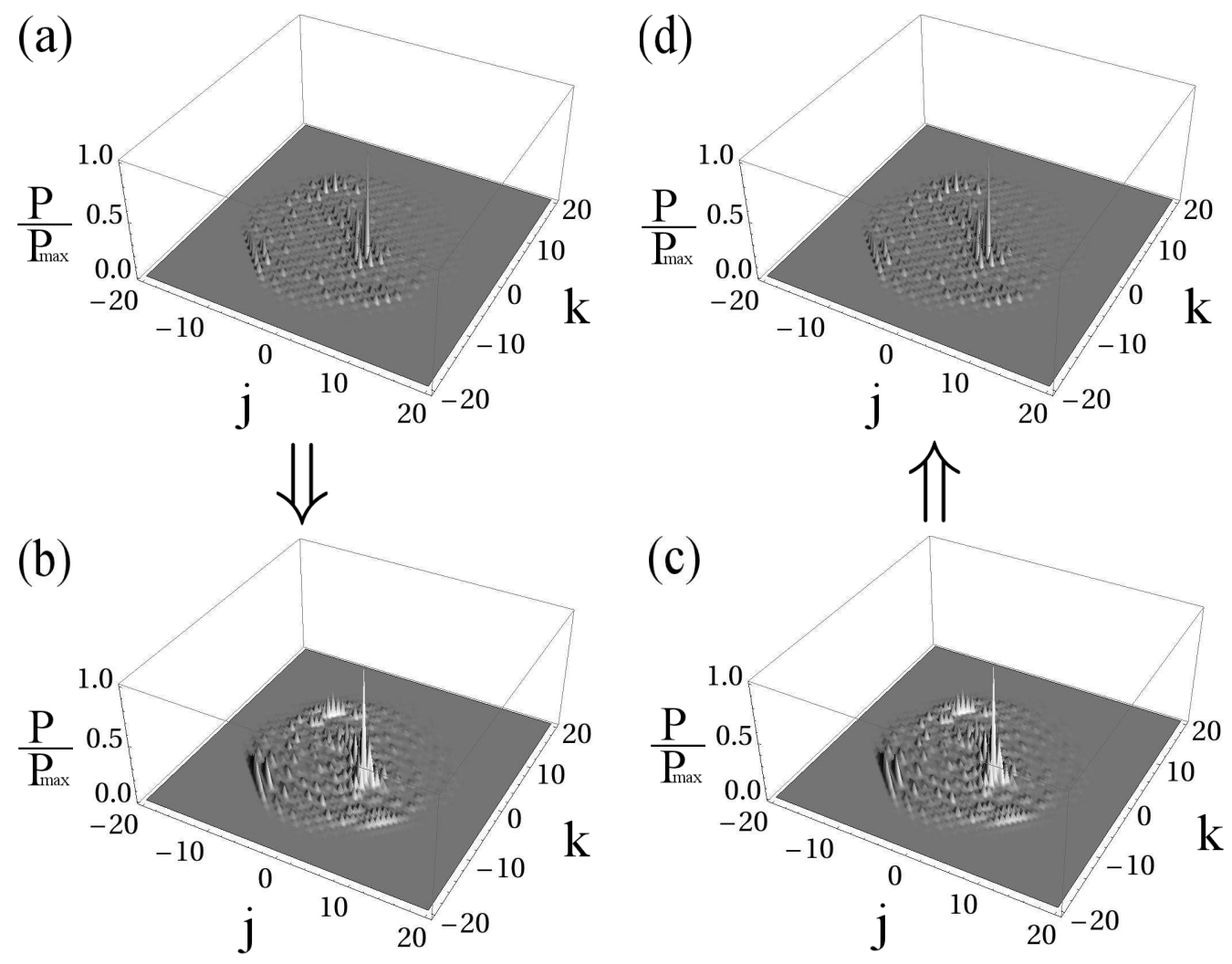

Figure 8. The same as in Fig. 5, but for the $4 \times 4$ DFT, Eq. (35).

defining the cross projectors. Furthermore, as expected the probabilities plots (not shown) are exactly the ones here, just rotated by $\pi / 4$ and rescaled by a factor $\sqrt{2}$.

\subsection{Quantum walks on a honeycomb lattice}

Our last example is a QW defined on a honeycomb lattice, whose structure is depicted in Fig. 9 (a). It is far more involving than the previous square case mainly because now the coordination number, equal to 3, is odd [47] (for coordination 3 in $1 \mathrm{D}$ see, e.g., Ref. [55]). We note that although this topology was recently investigated in the context of continuous time models [56], and few discrete time implementations do exist for torus-like boundary conditions [57] and for a similar three-state QW [58] (but not in a truly honeycomb geometry), the present is the first general treatment for the problem.

The labeling of the sites in both formulations is indicated in Fig. 9 (b). Without loss of generality, we adopt the convention: in the $y$-direction the quantum number $k$ is chosen such that if $k$ is even, then the corresponding infinite row of sites have the bonds along north (up), southeast and southwest. On the other hand, for $k$ odd, the bonds configuration at each site is south (down), northeast and northwest.

An interesting aspect of the honeycomb lattice, consequence of how it imposes the relation between the quantum number $\sigma$ and directions, is that any sequence:

$$
\left(j_{0}, k_{0}\right)_{\sigma} \stackrel{U}{\rightarrow}\left(j_{1}, k_{1}\right)_{\sigma} \stackrel{U}{\rightarrow}\left(j_{2}, k_{2}\right)_{\sigma} \ldots \stackrel{U}{\rightarrow}\left(j_{N-1}, k_{N-1}\right)_{\sigma} \stackrel{U}{\rightarrow}\left(j_{N}, k_{N}\right)_{\sigma}
$$




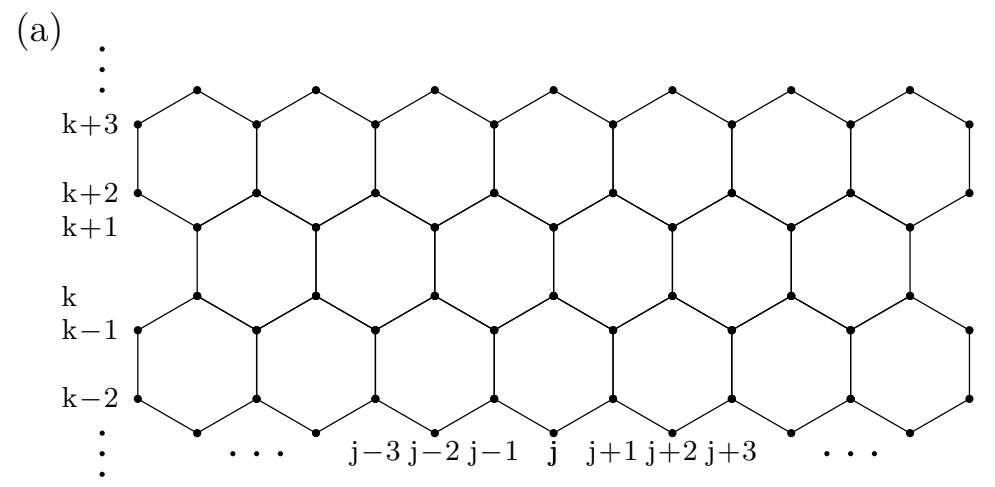

(b)

(c)

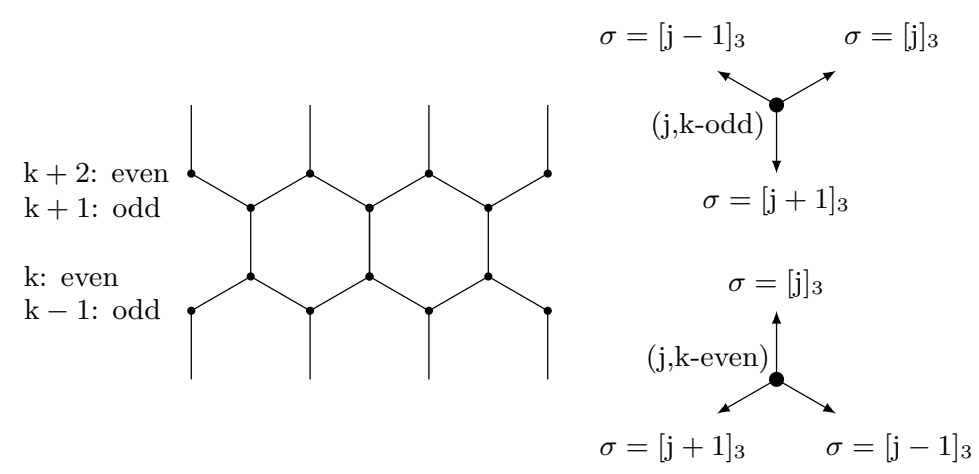

Figure 9. (a) The honeycomb lattice and (b) the convention for the sites labeling. (c) For the coined formulation, the association between the quantum number $\sigma$ and directions. Here $[x]_{3}$ denotes $x \bmod 3$, i.e., the remainder of $x / 3$.

i.e., a particular $N$ steps evolution for which the value of $\sigma$ remains the same, is not naturally (i.e., necessarily) a ballistic-like trajectory. By natural we mean those cases (like regular lattices with an even coordination number, e.g., our square lattice), where we always can associate quantum states and directions such that $\sigma \rightarrow \sigma$ leads to evolution along straight lines. In such cases, the above dynamics, Eq. (37), would be ballistic in the Hilbert lattice space regardless the specific $\sigma$. As we are going to see for the construction adopted here, successive transitions $\sigma \rightarrow \sigma$ yield a return to a same site (in a round trip) after visiting six sites. Hence, diffusion throughout the lattice implies transitions of the type $\sigma \rightarrow \sigma^{\prime} \neq \sigma$.

\subsubsection{The coined model}

For the inner coin states $|\sigma\rangle$, we have three possibilities, namely, $\sigma=0,1,2$ (we also could call them 1,2,3, but the use of 0 instead of 3 simplifies the notation). Due to the honeycomb particular topology, some care is necessary in defining the dynamics in terms of the coin variable. Indeed, states with a same $\sigma$ but at different sites does not always evolve to a same direction. So, to properly associate the $\sigma$ 's with the system evolution under $U$, we consider the following prescription (which, however, is not the 


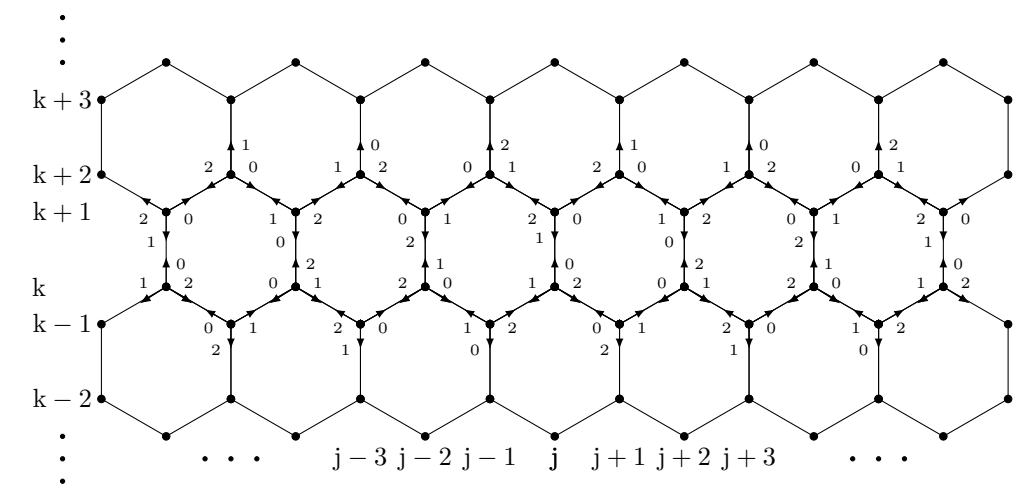

Figure 10. An illustration of the resulting association between coin quantum numbers and directions according to the rules in Fig. 9 (c). Here $k$ is even and $[j]_{3}=0$.

only possible $\ddagger)$. Let $[x]_{N} \equiv x \bmod N$, i.e., $[x]_{N}$ is the remainder of $x / N$. Then, for a given site $(j, k)$, the quantum numbers $0,1,2$ are associated to directions in the lattice as indicated in Fig. 9 (c). An example of the resulting configuration is displayed in Fig. 10 (in special, note from Fig. 10 that leaving from a certain site, say $(j, k)$, and always evolving to a same $\sigma$, mandatorily will get back to $(j, k)$ in exactly six steps). The rules in Fig. 9 (c) establish in an unique and self-consistent way - for the entire lattice - how single steps $(j, k) \rightarrow\left(j^{\prime}, k^{\prime}\right)$ are determined by the values of $\sigma$.

The basis states are written as $|(j, k)\rangle \otimes|\sigma\rangle$, with a column vector representation for $|\sigma\rangle$ given by

$$
|0\rangle=\left(\begin{array}{l}
1 \\
0 \\
0
\end{array}\right),|1\rangle=\left(\begin{array}{l}
0 \\
1 \\
0
\end{array}\right),|2\rangle=\left(\begin{array}{l}
0 \\
0 \\
1
\end{array}\right) .
$$

So, the coin operator at any site $(j, k)$ is

$$
C^{(j, k)}=\left(\begin{array}{ccc}
c_{00}^{(j, k)} & c_{01}^{(j, k)} & c_{02}^{(j, k)} \\
c_{10}^{(j, k)} & c_{11}^{(j, k)} & c_{12}^{(j, k)} \\
c_{20}^{(j, k)} & c_{21}^{(j, k)} & c_{22}^{(j, k)}
\end{array}\right)
$$

which we suppose to be an unitary matrix.

Assuming the above construction, after a little lengthy but straightforward analyzes one finds that the one step time evolution operator for the coined formulation reads

$$
U_{c}=\left(\sum_{\sigma=0}^{2} S_{\sigma} \otimes|\sigma\rangle\langle\sigma|\right) \times\left(\sum_{j, k}|(j, k)\rangle\langle(j, k)| \otimes C^{(j, k)}\right),
$$

where

$$
\begin{aligned}
& S_{\sigma}|(j, k)\rangle=|(f(j, k ; \sigma), g(j, k ; \sigma))\rangle, \\
& S_{\sigma}^{\dagger}|(j, k)\rangle=\left|\left(f\left(j, k ; \phi_{k}(\sigma)\right), g\left(j, k ; \phi_{k}(\sigma)\right)\right)\right\rangle,
\end{aligned}
$$

$\ddagger$ A full classification of equivalent constructions for the honeycomb lattice will appear elsewhere. 

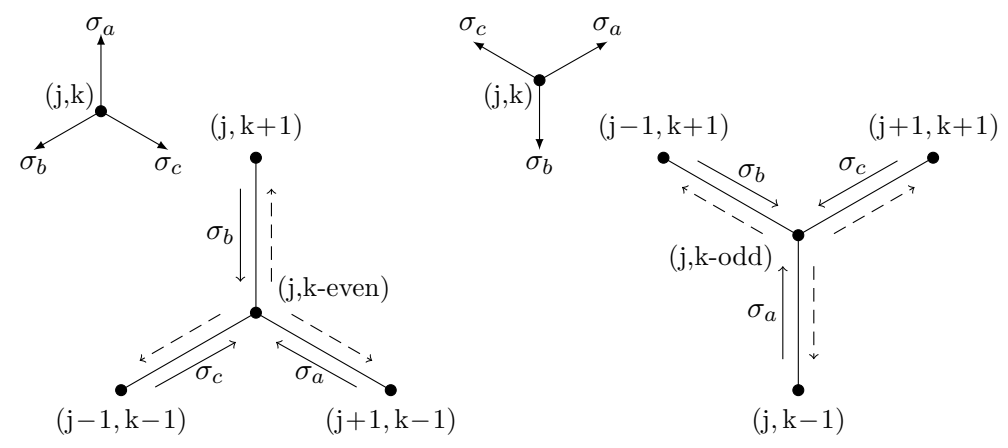

Figure 11. For the honeycomb lattice, the SQW basis states labeling rules. The $\sigma$ values here $\left(\sigma_{a}, \sigma_{b}\right.$ and $\left.\sigma_{c}\right)$ are defined in Eq. (44). The upper details summarize the convention in Fig. 9 (c) to label the CQW inner states.

for $($ with $\operatorname{sgn}[x]=x /|x|$, if $x \neq 0$, and $\operatorname{sgn}[0]=0)$

$$
\begin{aligned}
& f(j, k ; \sigma)=j+(-1)^{\sigma-\left[j+[k]_{2}\right]_{3}} \operatorname{sgn}\left[\sigma-\left[j+[k]_{2}\right]_{3}\right], \\
& g(j, k ; \sigma)=k+(-1)^{k}\left(1-2 \operatorname{sgn}\left[\left|\sigma-\left[j+[k]_{2}\right]_{3}\right|\right]\right), \\
& \phi_{k}(\sigma)=\left[\sigma-(-1)^{k}\right]_{3} .
\end{aligned}
$$

By construction $S_{\sigma} S_{\sigma}^{\dagger}=S_{\sigma}^{\dagger} S_{\sigma}=\mathbb{I}$, leading to an unitary $U_{c}$.

Finally, the corresponding projector operator is

$$
\mathcal{P}_{c}^{(j, k)}=|(j, k)\rangle\left\langle(j, k)\left|\otimes \sum_{\sigma=0}^{2}\right| \sigma\right\rangle\langle\sigma| .
$$

\subsubsection{The scattering model}

For the scattering formulation, we consider the schematics in Fig 11. We denote all the basis state incoming to the site $(j, k)$ (continuous arrows in Fig. 11) by $|\sigma,(j, k)\rangle$. Since now the lattice coordination is 3 , we need more than the usual \pm to label the direction quantum number $\sigma$. Therefore, we assume that $\sigma$ has the values $0,1,2$, in analogy with the coined model. But in each bond we can ascribe only two of these possible values to the two states representing opposite directions. In this way, for the general situation represented in Fig. 11, we set the convention (which can be checked to be self-consistent along the whole lattice)

$$
\sigma_{a}=[j]_{3}, \quad \sigma_{b}=[j+1]_{3}, \quad \sigma_{c}=[j-1]_{3} .
$$

At each bond, for a state $|\sigma,(j, k)\rangle$ incoming to $(j, k)$ there is a corresponding outgoing state, which is itself an incoming state to another site $\left(j^{\prime}, k^{\prime}\right)$ and denoted by $\left|\sigma^{\prime},\left(j^{\prime}, k^{\prime}\right)\right\rangle$ (dashed arrows in Fig. 11). From the protocol used to label the $\sigma^{\prime}$ s - Fig. 11 and Eq. (44) - it is not difficult to show that $\sigma^{\prime}=\phi_{k}(\sigma), j^{\prime}=f\left(j, k ; \phi_{k}(\sigma)\right)$, and $k^{\prime}=$ $g\left(j, k ; \phi_{k}(\sigma)\right)$, for $\phi, f$ and $g$ given in Eq. (42). For instance, in Fig. 11 for $k$ even and $[j]_{3}=0, \sigma_{a}=0$ and the other state in the same bond than $|0,(j, k)\rangle$ is $|2,(j+1, k-1)\rangle$, as verified by direct inspection. Likewise, this follows directly from $\sigma^{\prime}=\phi_{k-\text { even }}(0)=2$, $j^{\prime}=\left.f(j, k ; 2)\right|_{k-\text { even, }[j]_{3}=0}=j+1$ and $\left.k^{\prime}=g(j, k ; 2)\right)\left.\right|_{k-\text { even, }, j]_{3}=0}=k-1$. 
Thus, the evolution operator is $U_{s}=T+R$, with

$$
\begin{aligned}
& T|\sigma,(j, k)\rangle=\sum_{\alpha \neq \sigma} t_{\phi_{k}(\alpha), \sigma}^{(j, k)}\left|\phi_{k}(\alpha),\left(f\left(j, k ; \phi_{k}(\alpha)\right), g\left(j, k ; \phi_{k}(\alpha)\right)\right)\right\rangle, \\
& R|\sigma,(j, k)\rangle=r_{\phi_{k}(\sigma), \sigma}^{(j, k)}\left|\phi_{k}(\sigma),\left(f\left(j, k ; \phi_{k}(\sigma)\right), g\left(j, k ; \phi_{k}(\sigma)\right)\right)\right\rangle,
\end{aligned}
$$

where furthermore

$$
\begin{aligned}
& T^{\dagger}|\sigma,(j, k)\rangle=\sum_{\alpha \neq \phi_{k}(\sigma)}\left[t_{\sigma, \alpha}^{\left(f\left(j, k ; \phi_{k}(\sigma)\right), g\left(j, k ; \phi_{k}(\sigma)\right)\right)}\right]^{*}\left|\alpha,\left(f\left(j, k ; \phi_{k}(\sigma)\right), g\left(j, k ; \phi_{k}(\sigma)\right)\right)\right\rangle, \\
& R^{\dagger}|\sigma,(j, k)\rangle=\left[r_{\sigma, \phi_{k}(\sigma)}^{\left(f\left(j, k ; \phi_{k}(\sigma)\right), g\left(j, k ; \phi_{k}(\sigma)\right)\right)}\right]^{*}\left|\phi_{k}(\sigma),\left(f\left(j, k ; \phi_{k}(\sigma)\right), g\left(j, k ; \phi_{k}(\sigma)\right)\right)\right\rangle .
\end{aligned}
$$

Now, if we define

$$
\Gamma^{(j, k)}=\left(\begin{array}{ccc}
\Gamma_{00}^{(j, k)} & \Gamma_{01}^{(j, k)} & \Gamma_{02}^{(j, k)} \\
\Gamma_{10}^{(j, k)} & \Gamma_{11}^{(j, k)} & \Gamma_{12}^{(j, k)} \\
\Gamma_{20}^{(j, k)} & \Gamma_{21}^{(j, k)} & \Gamma_{22}^{(j, k)}
\end{array}\right),
$$

and identify $(\alpha, \beta=0,1,2)$

$$
r_{\phi_{k}(\sigma), \sigma}=\Gamma_{\phi_{k}(\sigma) \sigma}^{(j, k)}, \quad t_{\beta, \alpha}=\Gamma_{\beta \alpha}^{(j, k)}\left(\text { for } \beta \neq \phi_{k}(\alpha)\right),
$$

one has that the unitarity of $\Gamma$ in Eq. (47) guarantees that $U_{s}$ is also unitary.

Finally, the projector operator reads

$$
\begin{aligned}
P_{s}^{(j, k)}= & \left|\phi_{k}(\sigma),\left(f\left(j, k ; \phi_{k}(\sigma)\right), g\left(j, k ; \phi_{k}(\sigma)\right)\right)\right\rangle\left\langle\left(f\left(j, k ; \phi_{k}(\sigma)\right), g\left(j, k ; \phi_{k}(\sigma)\right)\right), \phi_{k}(\sigma)\right| \\
& +|\sigma,(j, k)\rangle\langle(j, k), \sigma| .
\end{aligned}
$$

\subsubsection{Mapping the models}

First, we note that: (i) comparing the $\sigma$ labeling convention for the coined and scattering formulation in the schematics in Fig. 11 (see also Eq. (44)); and (ii) taking into account how the states evolve according to the quantum number $\sigma$ in both models; it turns out that a direct one-to-one association between basis states is simply given by

$$
E|\sigma,(j, k)\rangle=|(j, k)\rangle \otimes|\sigma\rangle .
$$

Second, if as done in Sec. 3.1, we set the coin matrix Eq. (39) and the scattering coefficients in Eq. (47) to be equal, then we find that the corresponding expressions for $U_{s}$ and $U_{c}$, with $E$ given by Eq. (50), satisfies to Eq. (17).

Lastly, to obtain the probabilities of one model by means of the other, we define the cross operators as in Eq. (18), using the definitions in Eqs. (43), (49) and (50).

\subsubsection{Examples}

To illustrate the dynamics in a honeycomb topology, we again consider different coin (and equivalent scattering) matrices and calculate the QWs time evolutions. We analyze the following five different examples for the transition probabilities (assumed to be the same at all the lattice sites): 
(a) $3 \times 3$ typical unbiased operator

$$
C_{\text {unb }}=\frac{1}{\sqrt{3}}\left(\begin{array}{ccc}
-1 & \exp [-\pi i / 3] & \exp [-\pi i / 3] \\
\exp [-\pi i / 3] & -1 & \exp [-\pi i / 3] \\
\exp [-\pi i / 3] & \exp [-\pi i / 3] & -1
\end{array}\right)
$$

(b) $3 \times 3$ biased (and real) operator

$$
C_{b i a_{3}}=\frac{1}{3}\left(\begin{array}{ccc}
1 & 1-\sqrt{3} & 1+\sqrt{3} \\
1+\sqrt{3} & 1 & 1-\sqrt{3} \\
1-\sqrt{3} & 1+\sqrt{3} & 1
\end{array}\right)
$$

(c) $3 \times 3$ Discrete Hartley Transform (DHT) [59]

$$
C_{D H T_{3}}=\frac{1}{2 \sqrt{3}}\left(\begin{array}{ccc}
2 & 2 & 2 \\
2 & -1+\sqrt{3} & -1-\sqrt{3} \\
2 & -1-\sqrt{3} & -1+\sqrt{3}
\end{array}\right)
$$

(d) $3 \times 3$ Grover

$$
C_{G_{3}}=\frac{1}{3}\left(\begin{array}{ccc}
-1 & 2 & 2 \\
2 & -1 & 2 \\
2 & 2 & -1
\end{array}\right)
$$

(e) $3 \times 3$ Discrete Fourier Transform (DFT)

$$
C_{D F T_{3}}=\frac{1}{\sqrt{3}}\left(\begin{array}{ccc}
\exp [2 \pi i / 3] & 1 & \exp [-2 \pi i / 3] \\
1 & 1 & 1 \\
\exp [-2 \pi i / 3] & 1 & \exp [2 \pi i / 3]
\end{array}\right) .
$$

Few comments about the matrices above are in order. The first case, Eq. (51), is a typical (complex) unbiased operator since the coefficients have the same value for their modulus square, $\left|c_{\sigma^{\prime} \sigma}\right|^{2}=1 / 3$. Therefore, the resulting transition probabilities are equal to $1 / 3$. On the other hand, the second, Eq. (52) - here with all entries real numbers is strongly biased because the transition probabilities are very different (but obviously summing up to 1 ). For instance, for the scattering formulation, $k$-even and $[j]_{3}=0$ in Fig. 11 (cf. Eq. (48)), we have $\left|t_{0,0}\right|^{2}=1 / 9 \approx 0.11,\left|t_{1,0}\right|^{2}=2(2+\sqrt{3}) / 9 \approx 0.83$, $\left|r_{2,0}\right|^{2}=2(2-\sqrt{3}) / 9 \approx 0.06$. The discrete Hartley transform matrix [59], Eq. (53), although not usually considered in QWs, is an interesting example to study due to its usefulness in signal processing [60], furthermore always being real. The other two are just the $3 \times 3$ versions of the Grover and DFT. Finally, note there is not a $3 \times 3$ Hadamard matrix.

Assuming as the initial states

$$
|\Psi(0)\rangle_{s}=|1,(0,0)\rangle, \quad E|\Psi(0)\rangle_{s}=|\Psi(0)\rangle_{c}=|(0,0)\rangle \otimes|1\rangle,
$$

we evolve the models 20 time steps. The resulting probability patterns are displayed in Figs. 12-16. The graphs are organized exactly as done in the Sec. 3.1 .4 (Figs. 5-8). 

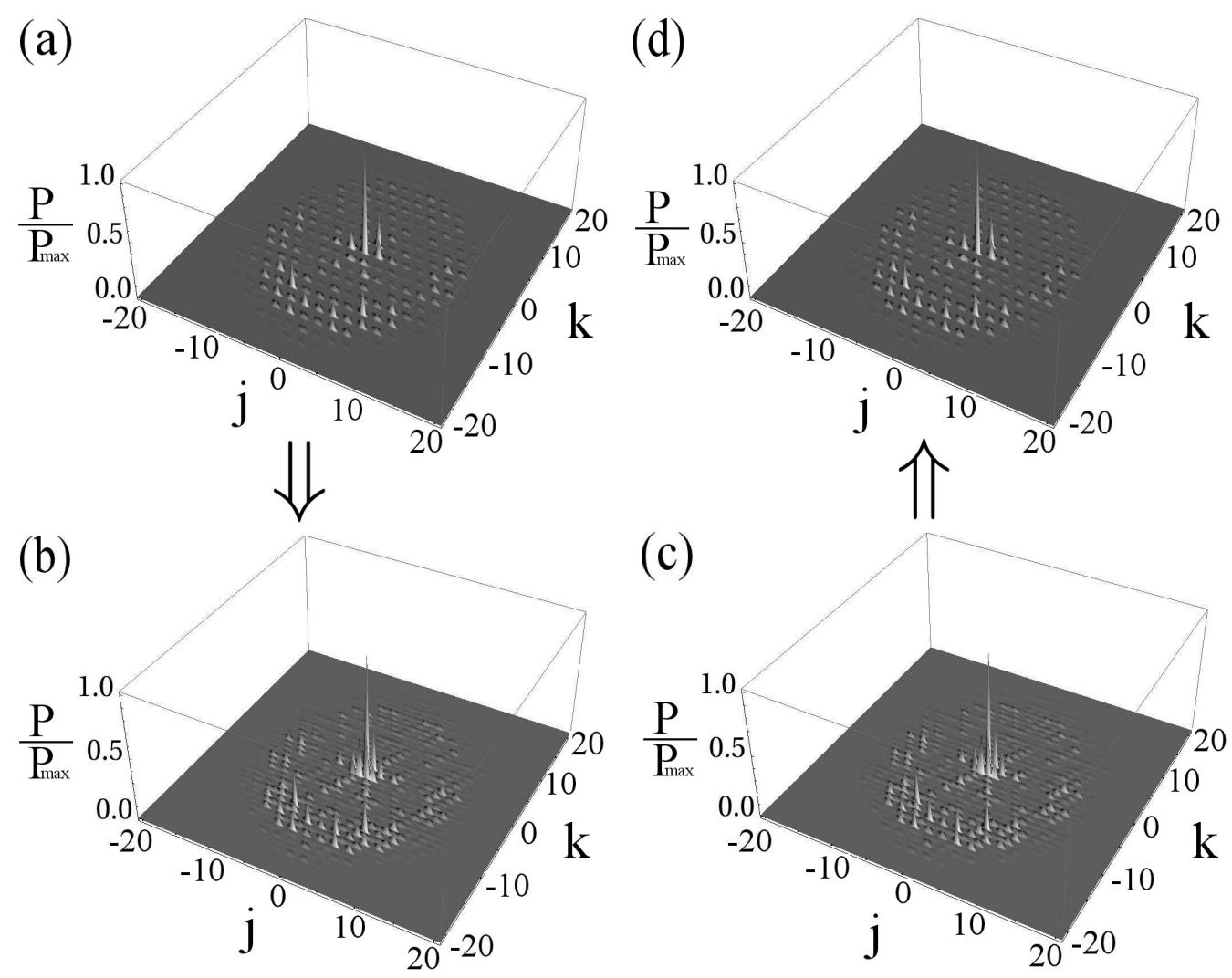

Figure 12. Quantum walks in the honeycomb lattice considering the $3 \times 3$ typical unbiased transition coefficients, Eq. (51). The density plots represent the probabilities to be in the different states (as defined by the projector operators) after $n=20$ time steps for $|\Psi(0)\rangle$ given in the main text. The results are for the: (a) coined, (b) scattering obtained from the coined, (c) scattering, and (d) coined obtained form the scattering, models. The graphs (a) and (d) and (b) and (c) are complete identical.

Again, certain facts are clearly observed from Figs. 12-16. First, as it should be, we can obtain one evolution from the other by correct projections. Second, like in the square lattice, in the honeycomb the CQW probabilities are in general more sparse and somewhat smoother than those for the SQW. And third, it is interesting to notice the particular pattern in Fig. 13, with a tendency of three preferable directions of propagation along the lattice. This is a consequence of the probabilities bias resulting from the matrix $C_{b i a_{3}}$, Eq. (52), for which three transition elements $c_{02}=c_{10}=c_{21}=(1+\sqrt{3}) / 3$ are considerable higher than the other six.

Finally, comparing the Grover (DFT) probability amplitudes for the square and honeycomb topologies, respectively, Figs. 7 and 15 (Figs. 8 and 16) we can have an idea on the influence of a regular lattice coordination number to the QW dynamics. In fact, note that the $|\Psi(0)\rangle$ used for the square lattice examples is equally "distributed" among the four $\sigma$ values basis states, Eq. (36), which is not the case for the honeycomb lattice whose $|\Psi(0)\rangle$ is written in terms of just one value of $\sigma$ (from the three possible), Eq. (56). Even then, we see that in the latter the resulting probabilities are more uniformly distributed, specially in the Grover case. This illustrates the intricate process 
Exemplifying the equivalence of discrete quantum walk models

(a)

(b)
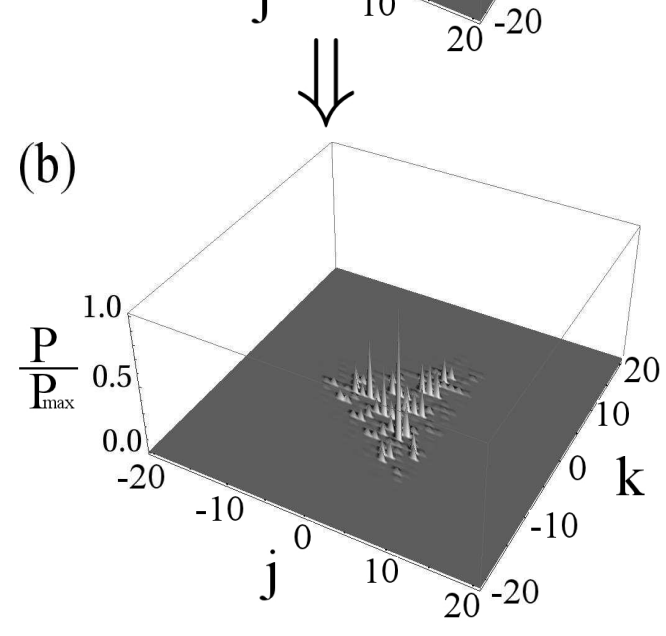

(d)

(c)

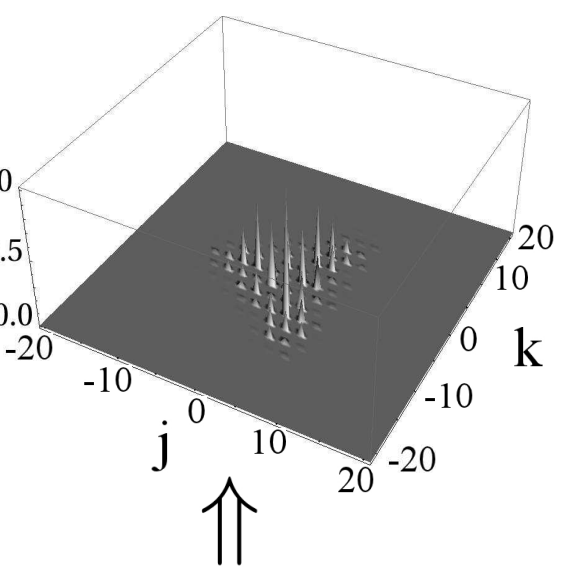

Figure 13. The same as in Fig. 12, but for the $3 \times 3$ biased real matrix, Eq. (52).
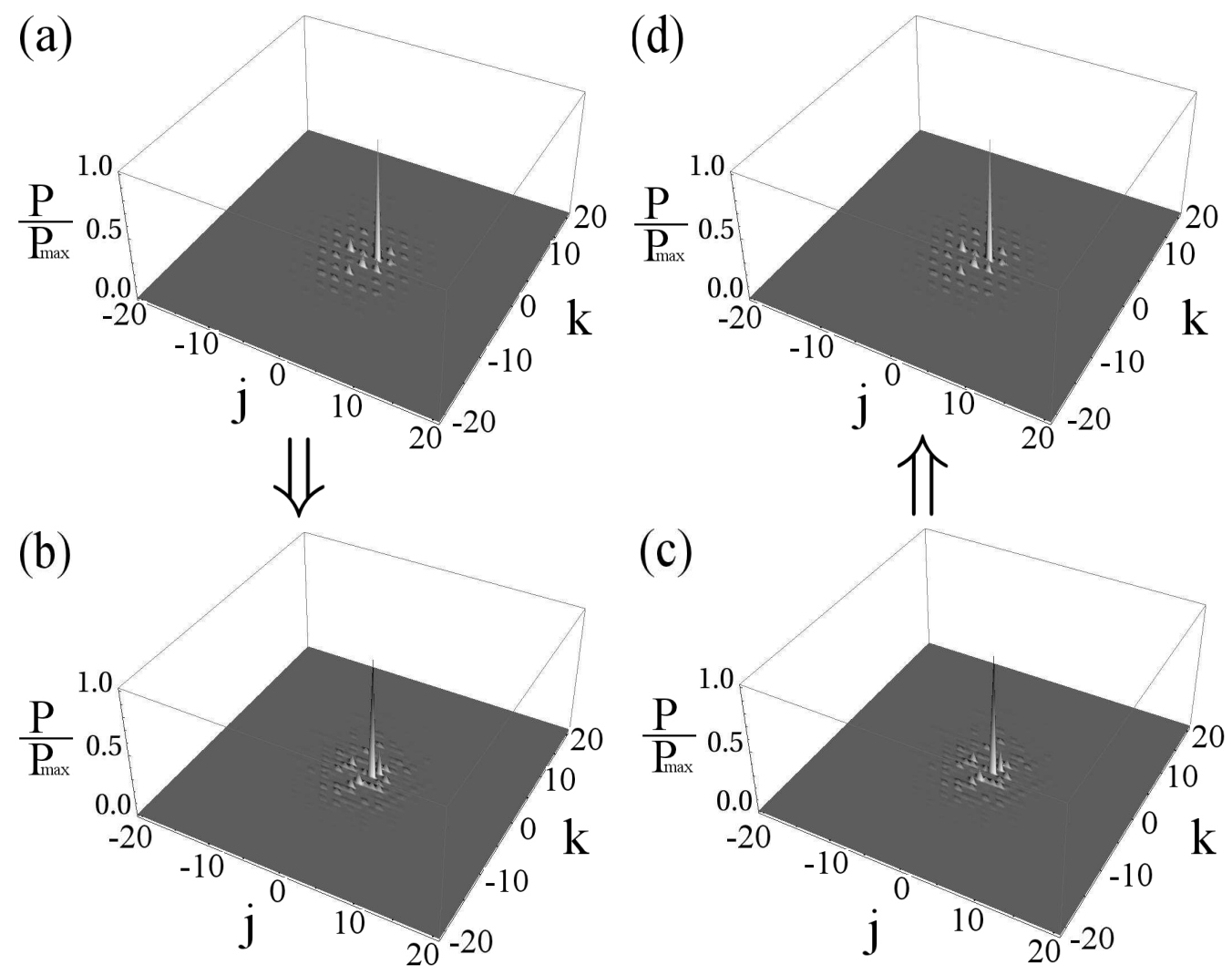

(c)
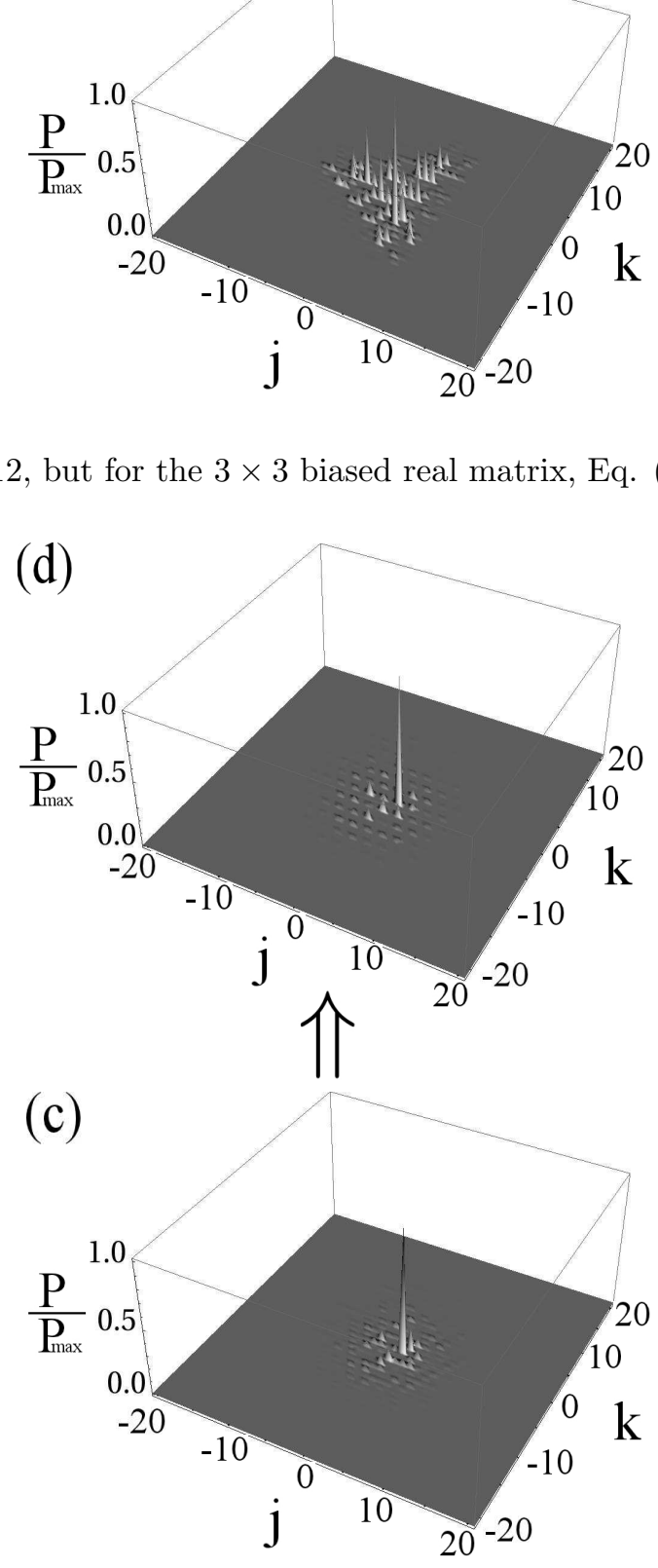

Figure 14. The same as in Fig. 12, but for the $3 \times 3$ DHT, Eq. (53). 
Exemplifying the equivalence of discrete quantum walk models

(a)

(b)


(d)

(c)



Figure 15. The same as in Fig. 12, but for the $3 \times 3$ Grover, Eq. (54).
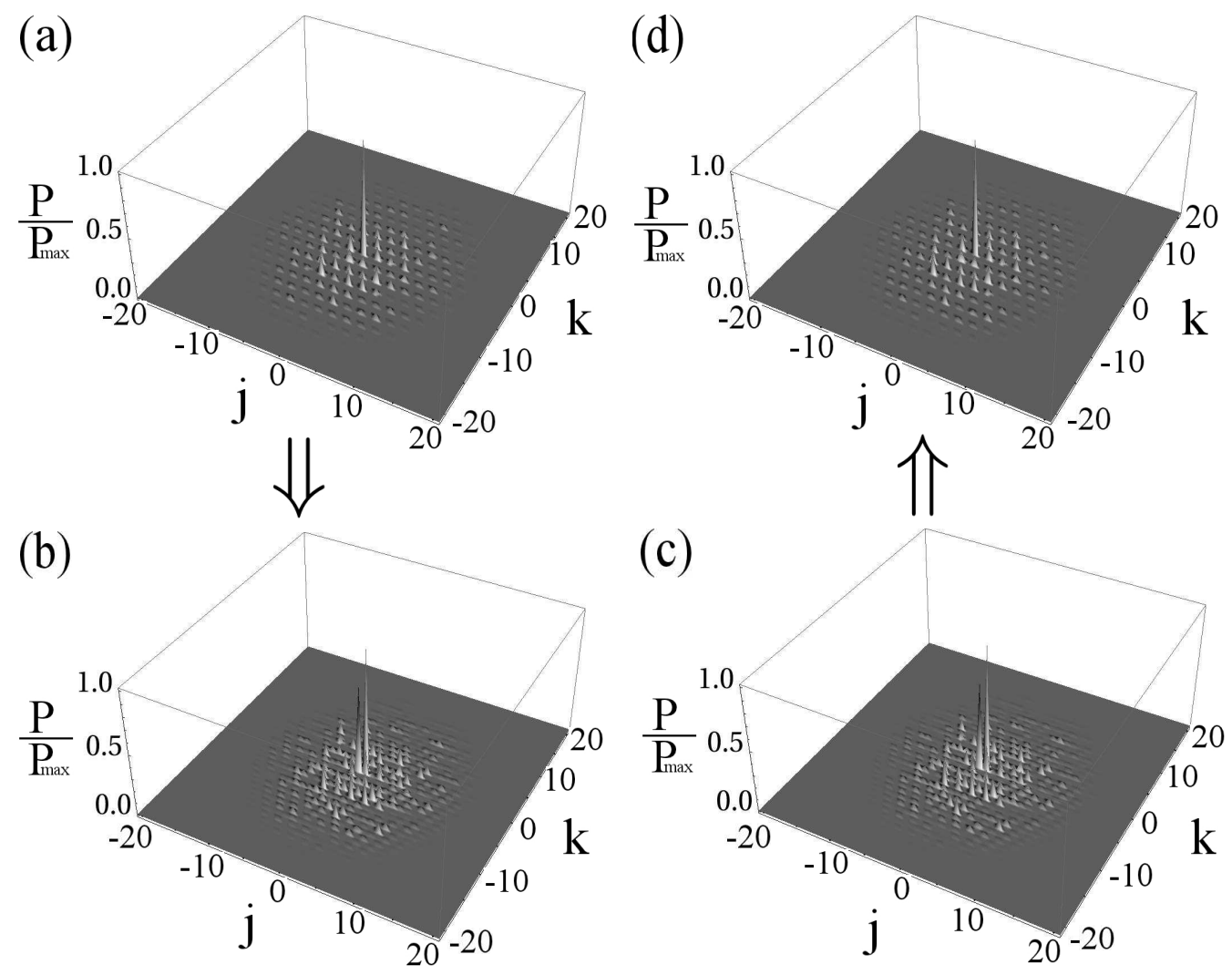

(c)
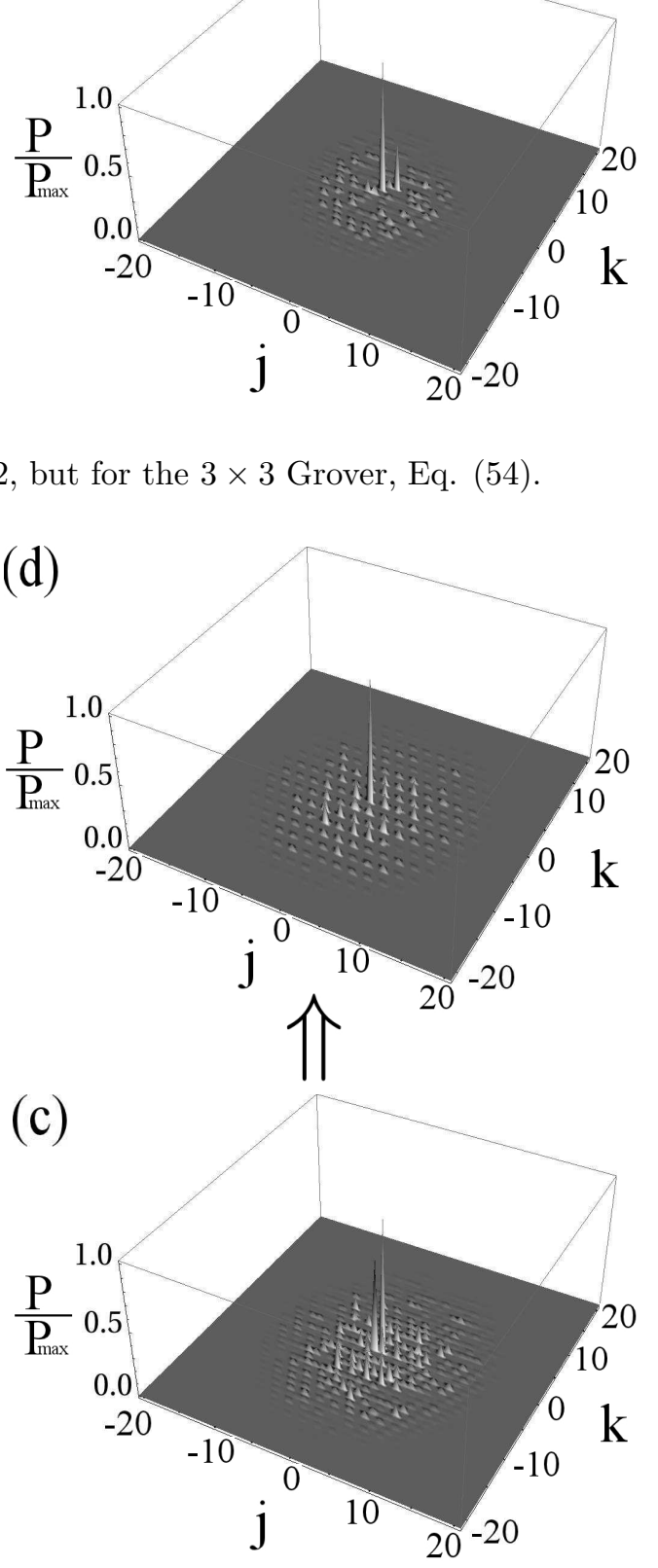

Figure 16. The same as in Fig. 12, but for the $3 \times 3$ DFT, Eq. (55). 
of quantum transitions at each lattice site as function of the number of bonds attached to it.

\section{Conclusion}

By means of detailed analysis of specific examples, in this work we have compared the different aspects associated to the construction of the discrete time coined and scattering quantum walks. Specifically, we have presented a complete formulation for the line, square and the involving honeycomb lattice topologies. In all the examples studied, we have clarified how to map one model version to the other. Also, we have illustrated the particularities in the probability pattern distributions along the graph structures, resulting from the different time evolution of each formulation. Finally, distinct coin (and corresponding scattering) matrices operators were considered. Besides the usual Hadamard, Grover and Discrete Fourier Transform, we have addressed the Discrete Hartley Transform as well as a few other cases.

Our aim here was to show through concrete situations that in fact the CQWs and SQWs are closely related. Moreover, the use of a specific QW version in different applications may be more a matter of practicality (or even of taste, as recently stressed [14]), than due to prohibitive conceptual differences between the models.

Regarding implementations, the issue may be a little subtle considering that usually distinct experimental architectures are proposed for CQWs and for SQWs (see the Introduction Sec.). However, a given realization of a specific QW model conceivably could also be used to obtain results from the other model. But then, the experimental setup somehow should allow the effective construction of proper cross projectors (as defined in Secs. 2-3 and 2-4). The important point is that efforts in this direction certainly would pay off since an actual implementation of one version of QWs having proper built-in projections to the other version - thus "carrying" the features of both type of QWs - would be much more flexible and useful in applications.

\section{Acknowledgements}

We are in great debt to M. C. Santos for help with the numerics. Research grants are provided by CNPq (da Luz) and Finepe-CTInfra.

\section{References}

[1] Aharonov Y, Davidovich L, and Zagury N 1993 Phys. Rev. A 481687

[2] Meyer D A 1996 J. Stat. Phys. 85551

[3] Watrous S 2001 J. Comput. Sys. Sci. 62376

[4] Kempe J 2003 Contemp. Phys. 44307

[5] Venegas-Andraca S E 2012 Quantum Information Processing 111015

[6] Farhi E and Gutmann S 1998 Phys. Rev. A 58915 Childs A M, Farhi E, and Gutmann S 2002 Quant. Info. Proc. 135

Childs A M, Cleve R, Deotto E, Farhi E, Gutmann S, and Spielman D A 2003 in Proceedings 
of the 35th ACM Symposium on Theory of Computing (STOC 2003) (ACM Press, New York), pp. $59-68$

[7] Tregenna B, Flanagan W, Maile R, and Kendon V 2003 New J. Phys. 583

[8] Hillery M, Bergou J, and Feldman E 2003 Phys. Rev. A 68032314

[9] Feldman E and Hillery M 2004 Phys. Lett. A 324277

[10] Feldman E and Hillery M 2007 J. Phys. A 4011343

[11] Shenvi S, Kempe J, Whaley K B 2003 Phys. Rev. A 67052307 Childs A M and Goldstone J 2004 Phys. Rev. A 70022314

[12] Gabris A, Kiss T, and Jex I 2007 Phys. Rev. A 76062315

[13] Hillery M, Reitzner D, and Buzek V 2010 Phys. Rev. A 81062324

[14] Hillery M, Zheng H, Feldman E, Reitzner D, and Buzek V 2012 Phys. Rev. A 85062325

[15] Kosik J and Busek V 2005 Phys. Rev. A 71012306

[16] Childs A M 2009 Phys. Rev. Lett. 102180501

[17] Karski M, Foerster L, Choi J-M, Steffen A, Alt W, Meschede D, and Widera A 2009 Science 325 174

[18] Leung G, Knott P, Bailey J, and Kendon V 2010 New J. Phys. 12123018

[19] Kempe J 2005 Probab. Theory Relat. Fields 133215

[20] Karafyllidis I G and Lagoudas D C 2007 Biosystems 88137

[21] Mohseni M, Rebentrost P, Loyd S, and Aspuru-Guzik A 2008 J. Chem. Phys. 129174106

Rebentrost P, Mohseni M, Kassal I, Loyd S, and Aspuru-Guzik A 2009 New J. Phys. 11033003

[22] Chandrashekar C M 2011 Phys. Rev. A 83022320

[23] Chandrashekar C M and Laflamme R 2008 Phys. Rev. A 78022314

[24] Ampadu A 2012 Commum. Theor. Phys. 5741

[25] Chandrashekar C M and Banerjee S 2011 Phys. Lett. A 3751553

[26] Aharonov D, Ambainis A, Kempe J, and Vazirani U 2001 in STOC'01: Proceedings of the 33nd Annual ACM Symposium on Theory of Computing (ACM, New York), pp. 37-49

Ambainis A, Bach E, Nayak A, Vishwanath A, and Watrous J 2001 in STOC'01: Proceedings of the 33nd Annual ACM Symposium on Theory of Computing (ACM, New York), pp. 50-59 Lovett N B, Cooper S, Everitt M, Trevers M, and Kendon V, 2010 Phys. Rev. A 81042330 Ambainis A 2008 in SOFSEM 2008: Theory and Practice of Computer Science, edited by Geffert V, Karhumaki J, Bertoni A, Preneel B, Navrat P, and Bielikova M (Springer, Berlin), pp. 1-4

Mosca M 2009 in Quantum Algorithms, Encyclopedia of Complexity Systems Science, edited by Meyers R A (Springer, Heidelberg)

[27] Dur W, Raussendorf R, Kendon V M, and Briegel H J 2002 Phys. Rev. A 66052319

[28] van Hoogdalem K A and Blaauboer M 2009 Phys. Rev. B 80125309

[29] Zou X, Dong Y, and Guo G 2006 New J. Phys. 881

[30] Tiegang D, Hillery M, and Zubairy M S 2004 Phys. Rev. A 70032304

[31] Crespi A, Sansoni L, Vallone G, Sciarrino F, Ramponi R, Mataloni P, and Osellame R 2012 in Frontiers in Ultrafast optics: biomedical, scientific, and industrial applications XII edited by Heisterkamp A, Meunier M, and Nolte S, Proc. of SPIE 8247, 82470L (Washington: SPIE Press)

[32] Perets H B, Lahini Y, Pozzi F, Sorel M, Morandotii R, and Silberberg Y 2008 Phys. Rev. Lett. 100170506

[33] Schreiber A, Cassemiro K N, Potocek V, Gabris A, Mosley P J, Andersson E, Jex I, and Silberhorn Ch 2010 Phys. Rev. Lett. 104050502

[34] Ryan C A, Laforest M, Boileau J C, and Laflamme R 2005 Phys. Rev. A 72062317

[35] Matjeschk R, Schneider CH, Enderlein M, Huber T, Schmitz H, Glueckert J, and Schaetz T 2012 New J. Phys. 14035012

[36] Jex I, Stenholm S, and Zeilinger A 1995 Opt. Commun. 11795

[37] Torma P and Jex I 1999 J. Opt. B 18 
[38] Do B, Stohler M L, Balasubramanian S, Elliott D S, Eash C, Fischbach E, Fischbach M A, Mills A, and Zwickl B 2005 J. Opt. Soc. Am. B 22499

[39] Strauch F W 2006 Phys. Rev. A $74030301(\mathrm{R})$

[40] Childs A M 2010 Commun. Math. Phys. 294581

[41] Andrade F M and da Luz M G E 2009 Phys. Rev. A 80052301

[42] Andrade F M and da Luz M G E 2011 Phys. Rev. A 84042343

[43] Ambainis A 2003 Int. J. Quant. Inform. 1507

[44] Ribeiro P, Milman P, and Mosseri R 2004 Phys. Rev. Lett. 93190503

[45] Nielsen M A and Chuang I L 2000 Quantum computation and quantum information (Cambridge: Cambridge Univ. Press)

[46] Bach E, Coppersmith S, Goldschen M P, Joynt R, and Watrous J 2004 J. Comput. Syst. Sci. 69 562

[47] Carneiro I, Loo M, Xu X, Girerd M, Kendon V, and Knight P L 2005 New J. Phys. 7156

[48] Chandrashekar C M, Srikanth R, and Laflamme R 2008 Phys. Rev. A 77, 032326

[49] da Luz M G E, Cheng B K, and Heller E J 1998 J. Phys. A 312975

Schmidt A G M, Cheng B K, and da Luz M G E 2002 Phys. Rev. A 66062712

Schmidt A G M, Cheng B K, and da Luz M G E 2003 J. Phys. A 36 L545

Zanetti F M, Kuhn J, Delben G J, Cheng B K, and da Luz M G E 2006 J. Phys. A 392493

[50] Chadan K and Sabatier P C 1989 Inverse problems in quantum scattering theory 2nd. edition (Berlin: Springer-Verlag)

[51] Andrade F M and da Luz M G E 2012 Phys. Rev. A 86042309

[52] Mackay T D, Bartlett S D, Stephenson L T, and B C Sanders 2002 J. Phys. A 352745

[53] Carneiro I, Loo M, Xu X, Girerd M, Kendon V, and Knight P L 2005 New J. Phys. 7156

[54] Oliveira A C, Portugal R, and Donangelo R 2006 Phys. Rev. A 74012312

[55] Inui N, Konno N, and Segawa E 2005 Phys. Rev. E 72056112

[56] Jafarizadeh M A and Sufiani R 2007 Physica A 381116

[57] Abal G, Donangelo R, Marquezinho F L, and Portugal R 2010 Math. Struct. Comput. Sci. 20 999

[58] Kollar B, Stefanak M, Kiss T, and Jex I 2010 Phys. Rev. A 82012303

[59] Ersoy O K 1994 Proceedings IEEE 82429

[60] Boussakta S, Alshibami O H, and Aziz M Y 2001 IEEE Trans. Signal Process. 493145 